\title{
Effects of Schwarzschild Black Hole Horizon on Isothermal Plasma Wave Dispersion
}

\author{
M. Sharif *and Umber Sheikh \\ Department of Mathematics, \\ University of the Punjab, Lahore 54590, Pakistan
}

\begin{abstract}
The 3+1 GRMHD equations for Schwarzschild spacetime in Rindler coordinates with isothermal state of plasma are formulated. We consider the cases of non-rotating and rotating backgrounds with non-magnetized and magnetized plasmas. For these cases, the perturbed form of these equations are linearized and Fourier analyzed by introducing plane wave type solutions. The determinant of these equations in each case leads to two dispersion relations which give value of the wave number $k$. Using the wave number, we obtain information like phase and group velocities etc. which help to discuss the nature of the waves and their characteristics. These provide interesting information about the black hole magnetosphere near the horizon. There are cases of normal and anomalous dispersion. We find a case of normal dispersion of waves when the plasma admits the properties of Veselago medium. Our results agree with those of Mackay et al. according to which rotation of a black hole is required for negative phase velocity propagation.
\end{abstract}

Keywords : $3+1$ formalism, GRMHD, Rindler spacetime, isothermal plasma.

\section{Introduction}

General Relativity (GR) is a beautiful scheme for describing the gravitational field and the corresponding equations. This theory is believed to apply to all forms of interaction whether these are between the charged particles or large scale gravitational structures, including compact objects like black holes. Black holes are still mysterious [1. While physicists have been grappling the theory of black holes, astronomers have been searching for real-life examples of black holes in the universe [2. It is proved that black holes exist on the basis of study of effects which they exert on their surroundings. With their enormous gravitational fields, they greatly affect the surrounding plasma medium (which is highly magnetized) and hence plasma physics in the vicinity of a black hole has become a subject of interest in astrophysics. In the immediate vicinity of a black hole, GR applies and it is therefore of interest to formulate plasma physics problem in this context.

In large scale gravitational structures, one often encounters magnetic fields. All the massive stars, neutron stars and black holes carry energy flux which produces a relatively large magnetic field. In order to understand such phenomena, we have a developed theory of magnetized plasma called theory of magnetohydrodynamics (MHD). In this theory, perfect MHD flow is important which is the flow of plasma with negligible charge separation and with a magnetic field frozen into it [3. Since all the compact objects have strong gravitational fields near their surfaces [4, it is important to study the general relativistic effects on physical processes taking place in their vicinity. The MHD theory with the effects of gravity is called general relativistic magnetohydrodynamics (GRMHD). The GRMHD equations include Maxwell's equations, Ohm's law, mass, momentum and energy conservation equations. These help us to study stationary configurations and dynamic evolution of conducting fluid in a magnetosphere. They are also required to investigate various aspects of the interaction of relativistic gravity with plasma's magnetic field.

The general relativistic physics of black holes and plasmas takes place in $3+1$ dimensional space while the corresponding relativistic laws are written in three-dimensional language. This formalism is well-suited to carry non-relativistic intuition of physicists about plasmas, hydrodynamics and stellar dynamics into the arena of

*msharif@math.pu.edu.pk 
black holes and GR. The formalism (also called ADM formalism) was originally developed by Arnowitt et al. [5] and was motivated by several startling results proved in 1970 using the black hole viewpoint [6]-[11]. Thorne and Macdonald [12-14 extended the formulation to electromagnetic fields of the black hole theory. The wave propagation theory in the Friedmann universe was investigated using $3+1$ formalism by Holcomb and Tajima [15], Holcomb [16] and Dettmann et al. [17. Khanna [18] derived the MHD equations describing the two component plasma theory of the Kerr black hole in this split. Antón et al. [19] used the formalism to investigate various test simulations and discussed magneto-rotational instability of accretion disks. Komissarov 20] discussed Blendford-Znajec monopole solution using $3+1$ formalism in black hole electrodynamics. Zhang [21]-22] formulated the black hole theory for stationary symmetric GRMHD using this formalism with its applications in Kerr geometry.

The gravitational perturbations away from the Schwarzschild background have been discussed by Regge and Wheeler [23. This treatment was extended by Zerilli [24] who showed that the perturbations corresponding to a change in mass, the angular momentum and charge of the Schwarzschild black hole are well-behaved. The decay of non well-behaved perturbations has been investigated by Price 25. The quasi-static electric problem was solved by Hanni and Ruffini 8 who proved that the lines of force diverge at the horizon for the observer at infinity. Wald [26] derived the solution for electromagnetic field occurring when a stationary axisymmetric black hole is placed in a uniform magnetic field aligned along the symmetry axis of black hole. A linearized treatment of plasma waves for special relativistic formulation of the Schwarzschild black hole was developed by Sakai and Kawata [27]. Using 3+1 ADM formalism, Buzzi et al. 28] extended this treatment to waves in general relativistic two component plasma propagating in radial direction. They investigated the one dimensional radial propagation of transverse and longitudinal waves close to the Schwarzschild horizon. In a recent paper, Sharif and Umber 29] have found some interesting properties of cold plasma in the vicinity of a Schwarzschild black hole horizon using $3+1$ GRMHD equations. They have also verified that the rotation is a cause of negative phase velocity propagation which coincides with the result of Mackay et al. [30].

The aim of this paper is to discuss the properties of waves in isothermal plasma. We develop and expound a theoretical method relevant to the study of relativistic fluid model used in applications to astrophysics and plasma physics. We consider Schwarzschild spacetime in Rindler coordinates which gives an example of the essential ideas of the horizon and the $3+1$ split without distracting complications of curved spacial three geometry. This investigation would lead to support the viewpoint that information from a black hole cannot be extracted.

The GRMHD equations in 3+1 formalism using isothermal plasma in the Schwarzschild magnetosphere are obtained. The component form of the equations is extracted by involving linear perturbations in each of the cases of either non-magnetized or magnetized, non-rotating or rotating perfect fluid. The condition for the plane harmonic wave is imposed on all the perturbations. The equations are then Fourier analyzed and solved to get the dispersion relations with the help of software Mathematica. Two dispersion relations are found in each case and the wave numbers are deduced uniquely in terms of angular frequency. The wave velocity, group velocity, refractive index and the corresponding quantities give the properties of plasma near the horizon expressed by the perfect fluid. These properties are shown in terms of graphs.

The scheme of the paper is as follows. The next section contains basic informatory results for $3+1$ formalism. The results for the GRMHD equation are given and energy equation is obtained. These equations are restricted for the case of perfect fluid with perfect MHD flow assumption for Rindler approximation of the Schwarzschild spacetime. The equations are further modified by adding the condition of isothermal plasma. Section 3 is devoted to the case of non-rotating background for which we find the dispersion relations. Section 4 is furnished with the values of $k$ for rotating non-magnetized plasma. In section 5, we generalize the problem to the case of rotating magnetized plasma. In the last section, we shall summarize and discuss the results.

\section{$2 \quad 3+1$ Spacetime Modeling}

The astrophysical theory in early 1960s was dominated by the frozen star viewpoint [31. As long as the viewpoint prevailed, physicists failed to realize that the black hole can be dynamical, evolving, energy-releasing object. Many problems of current interest in black-hole astrophysics cannot be posed in frozen-star viewpoint, because the viewpoint has difficulty producing unambiguous boundary conditions at the event horizon. Such problems where it is inadequate to replace the black hole horizon, in picture and calculations, by a surface of no return, a new viewpoint for the black hole physics was developed - the membrane paradigm (a marriage 
of horizon formalism with $3+1$ formalism). This is mathematically equivalent to the standard, full, general relativistic theory of black holes for the physics outside the horizon. Although this formalism is not treated in standard relativity textbooks, it has been developed in great detail by many researchers and has played an important role [14.

In $3+1$ formalism, the line element of the spacetime can be written as 22$]$

$$
d s^{2}=-\alpha^{2} d t^{2}+\gamma_{i j}\left(d x^{i}+\beta^{i} d t\right)\left(d x^{j}+\beta^{j} d t\right)
$$

where $\alpha$ is the lapse function, $\beta^{i}$ are the components of shift vector and $\gamma_{i j}$ are the components of the spatial metric. All these quantities are functions of the coordinates $t, x^{i}$. A natural observer, associated with this spacetime called fiducial observer (FIDO), has four-velocity $\mathbf{n}$ perpendicular to the hypersurfaces of constant time $t$. Notice that we are using geometrized units.

The Rindler approximation of the Schwarzschild line element is [14]

$$
d s^{2}=-\alpha^{2}(z) d t^{2}+d x^{2}+d y^{2}+d z^{2}
$$

which is an analogue of the Schwarzschild spacetime with $z, x$ and $y$ as radial $r$, axial $\phi$ and poloidal $\theta$ directions respectively. In Rindler coordinates, the lapse function $\alpha$ is $\frac{z}{2 r_{H}}$, where $r_{H}$ is the radius of the Schwarzschild black hole. This function clearly vanishes at the horizon which we can place at $z=0$. It is useful to think of this absolute space with fiducial observer which never moves from its fixed location. The relationship between the universal time $t$ and FIDO measured time $\tau$ can be expressed by the time lapse $\alpha \equiv \frac{d \tau}{d t}$. The Schwarzschild black hole is non-rotating, hence the shift vector $\beta=0$. This is the minimum configuration of the Kerr black hole out of which no energy can be extracted.

\subsection{Perfect GRMHD Equations}

In magnetohydrodynamic treatment, the plasma is represented as a perfect fluid. The perfect MHD flow assumption is given by

$$
\mathbf{E}+\mathbf{V} \times \mathbf{B}=0,
$$

where $\mathbf{V}, \mathbf{B}$ and $\mathbf{E}$ are the velocity, magnetic and electric fields of the fluid measured by FIDO. This assumption shows that fluid possesses no electric field in rest-frame and the electric field is perpendicular to the magnetic field.

It is known that unlike cold plasma approximation, isothermal plasma contains plasma pressure which cannot be ignored. The pressure gradient term is to be included. In this model, the energy equation is also introduced but the term involving the heat flux is neglected. Because of the vanishing thermal conductivity, the plasma is non-viscous and consequently the non-diagonal terms of the pressure dyad are all equal to zero. FIDO measured local energy conservation law 21] is given by

$$
\frac{d \epsilon}{d \tau}+\theta \epsilon+\frac{1}{2 \alpha} W^{i j}\left(£_{t} \gamma^{i j}\right)=-\frac{1}{\alpha^{2}} \nabla \cdot\left(\alpha^{2} \mathbf{S}\right)+\frac{\mathbf{1}}{\alpha} \nabla \beta: \overleftrightarrow{\mathbf{W}}+\mathbf{E} \cdot \mathbf{j} .
$$

Here $\epsilon, \mathbf{S}, W^{i j}$ and $\mathbf{j}$ represent the mass energy density, energy flux, stress tensor and current vector of the electromagnetic field in three-dimensions respectively, $\theta$ is the expansion rate of the FIDO's four-velocity, $£_{t}$ is the time derivative along shifting congruence (Lie derivative with respect to global time in a usual fashion) and $\frac{d}{d \tau} \equiv \frac{1}{\alpha}\left(\frac{\partial}{\partial t}-\beta . \nabla\right)$ is the rate of change of a three-dimensional vector which lies in the absolute space according to the FIDO. When we substitute the following values for perfect fluid 12 .

$$
\begin{aligned}
\epsilon & =\left(\mu \rho_{0}-p\left(1-\mathbf{V}^{2}\right)\right) \gamma^{2} \\
\mathbf{S} & =\mu \rho_{0} \gamma^{2} \mathbf{V} \\
\overleftrightarrow{\mathbf{W}} & =\mu \rho_{0} \gamma^{2} \mathbf{V} \otimes \mathbf{V}+p \overleftrightarrow{\gamma}
\end{aligned}
$$

with $\mu$ the specific enthalpy, $\rho_{0}$ is the rest-mass density, $p$ is the pressure, and perfect MHD condition, Eq.(2.3) 
takes the following form

$$
\begin{aligned}
& \rho_{0} \gamma^{2} \frac{D \mu}{D \tau}+\mu \gamma^{2} \frac{D \rho_{0}}{D \tau}+2 \rho_{0} \mu \gamma^{4} \mathbf{V} \cdot \frac{D \mathbf{V}}{D \tau}-\frac{d p}{d \tau}+\left(\mu \rho_{0} \gamma^{2}-p\right) \theta+2 \rho_{0} \mu \gamma^{2} \mathbf{V} \cdot \mathbf{a}+\rho_{0} \mu \gamma^{2}(\nabla \cdot \mathbf{V}) \\
& -\frac{1}{\alpha}\left\{\rho_{0} \mu \gamma^{2} \mathbf{V} \cdot(\mathbf{V} \cdot \nabla) \beta+p(\nabla \cdot \beta)\right\}+\frac{1}{2 \alpha}\left\{\rho_{0} \mu \gamma^{2} V^{i} V^{j}+p \gamma^{i j}\right\} £_{t} \gamma_{i j}+\frac{1}{4 \pi \alpha}[(\mathbf{V} \times \mathbf{B}) \cdot(\nabla \times(\alpha \mathbf{B})) \\
& \left.+\alpha(\mathbf{V} \times \mathbf{B}) \cdot \frac{d}{d \tau}(\mathbf{V} \times \mathbf{B})+(\mathbf{V} \times \mathbf{B}) \cdot(\mathbf{V} \times \mathbf{B} \cdot \nabla) \beta+\theta \alpha(\mathbf{V} \times \mathbf{B}) \cdot(\mathbf{V} \times \mathbf{B})\right]=0,
\end{aligned}
$$

where

$$
\frac{D}{D \tau} \equiv \frac{d}{d \tau}+\mathbf{V} . \nabla=\frac{1}{\alpha}\left\{\frac{\partial}{\partial t}+(\alpha \mathbf{V}-\beta) . \nabla\right\} .
$$

For the Schwarzschild black hole, FIDO measured Faraday's law, equation of evolution of the magnetic field, local conservation laws of mass and momentum are given by Eqs.(2.17)-(2.20) [29. These equations along with local conservation law of energy Eq.(2.4) become

$$
\begin{aligned}
& \frac{\partial \mathbf{B}}{\partial t}=\nabla \times(\alpha \mathbf{V} \times \mathbf{B}) \\
& \frac{\partial \mathbf{B}}{\partial t}+(\alpha \mathbf{V} \cdot \nabla) \mathbf{B}-(\mathbf{B} \cdot \nabla)(\alpha \mathbf{V})+(\nabla \cdot(\alpha \mathbf{V})) \mathbf{B}=0 \\
& \frac{\partial\left(\rho_{0} \mu\right)}{\partial t}+\{(\alpha \mathbf{V}) \cdot \nabla\}\left(\rho_{0} \mu\right)+\rho_{0} \mu \gamma^{2} \mathbf{V} \cdot \frac{\partial \mathbf{V}}{\partial t}+\rho_{0} \mu \gamma^{2} \mathbf{V} \cdot(\alpha \mathbf{V} \cdot \nabla) \mathbf{V}+\rho_{0} \mu\{\nabla \cdot(\alpha \mathbf{V})\}=0 \\
& \left\{\left(\rho_{0} \mu \gamma^{2}+\frac{\mathbf{B}^{2}}{4 \pi}\right) \delta_{i j}+\rho_{0} \mu \gamma^{4} V_{i} V_{j}-\frac{1}{4 \pi} B_{i} B_{j}\right\}\left(\frac{1}{\alpha} \frac{\partial}{\partial t}+\mathbf{V} \cdot \nabla\right) V^{j}-\left(\frac{\mathbf{B}^{2}}{4 \pi} \delta_{i j}-\frac{1}{4 \pi} B_{i} B_{j}\right) V_{, k}^{j} V^{k} \\
& +\rho_{0} \gamma^{2} V_{i}\left\{\frac{1}{\alpha} \frac{\partial \mu}{\partial t}+(\mathbf{V} \cdot \nabla) \mu\right\}=-\rho_{0} \mu \gamma^{2} a_{i}-p_{, i}+\frac{1}{4 \pi}(\mathbf{V} \times \mathbf{B})_{i} \nabla \cdot(\mathbf{V} \times \mathbf{B})-\frac{1}{8 \pi \alpha^{2}}(\alpha \mathbf{B})_{, i}^{2} \\
& +\frac{1}{4 \pi \alpha}\left(\alpha B_{i}\right)_{, j} B^{j}-\frac{1}{4 \pi \alpha}[\mathbf{B} \times\{\mathbf{V} \times(\nabla \times(\alpha \mathbf{V} \times \mathbf{B}))\}]_{i}, \\
& \gamma^{2}\left(\frac{1}{\alpha} \frac{\partial}{\partial t}+\mathbf{V} \cdot \nabla\right)\left(\mu \rho_{0}\right)-\frac{1}{\alpha} \frac{\partial p}{\partial t}+2 \rho_{0} \mu \gamma^{4} \mathbf{V} \cdot\left(\frac{1}{\alpha} \frac{\partial}{\partial t}+\mathbf{V} \cdot \nabla\right) \mathbf{V}+2 \rho_{0} \mu \gamma^{2}(\mathbf{V} \cdot \mathbf{a}) \\
& +\rho_{0} \mu \gamma^{2}(\nabla \cdot \mathbf{V})+\frac{1}{4 \pi \alpha}\left[(\mathbf{V} \times \mathbf{B}) \cdot(\nabla \times(\alpha \mathbf{B}))+(\mathbf{V} \times \mathbf{B}) \cdot \frac{\partial}{\partial t}(\mathbf{V} \times \mathbf{B})\right]=0
\end{aligned}
$$

where $\mathbf{a}$ is the gravitational acceleration. Notice that here Eq.(2.4) is the new equation in addition to Eqs.(2.17)(2.20) 29. These constitute the perfect GRMHD equations for fluid with non-zero pressure in the vicinity of a black hole horizon.

\subsection{GRMHD Equations for Isothermal Plasma in Schwarzschild Spacetime}

The isothermal equation of state can be expressed by the following equation [21]

$$
\mu=\frac{\rho+p}{\rho_{0}}=\text { constant } .
$$

Here $\rho$ is the mass density and pressure of the fluid.

For the Rindler approximation of Schwarzschild geometry, FIDOs four-velocity expansion rate and shift vector vanish. Thus the perfect GRMHD equations in $3+1$ formalism for isothermal state of plasma turn out 
to be

$$
\begin{aligned}
& \frac{\partial \mathbf{B}}{\partial t}=\nabla \times(\alpha \mathbf{V} \times \mathbf{B}) \\
& \frac{\partial \mathbf{B}}{\partial t}+(\alpha \mathbf{V} \cdot \nabla) \mathbf{B}-(\mathbf{B} \cdot \nabla)(\alpha \mathbf{V})+\mathbf{B} \nabla \cdot(\alpha \mathbf{V})=0 \\
& \frac{\partial(\rho+p)}{\partial t}+(\alpha \mathbf{V} \cdot \nabla)(\rho+p)+(\rho+p) \gamma^{2} \mathbf{V} \cdot \frac{\partial \mathbf{V}}{\partial t}+(\rho+p) \gamma^{2} \mathbf{V} \cdot(\alpha \mathbf{V} \cdot \nabla) \mathbf{V}+(\rho+p) \nabla \cdot(\alpha \mathbf{V})=0 \\
& \left\{\left((\rho+p) \gamma^{2}+\frac{\mathbf{B}^{2}}{4 \pi}\right) \delta_{i j}+(\rho+p) \gamma^{4} V_{i} V_{j}-\frac{1}{4 \pi} B_{i} B_{j}\right\}\left(\frac{1}{\alpha} \frac{\partial}{\partial t}+\mathbf{V} \cdot \nabla\right) V^{j} \\
& -\left(\frac{\mathbf{B}^{2}}{4 \pi} \delta_{i j}-\frac{1}{4 \pi} B_{i} B_{j}\right) V_{, k}^{j} V^{k}=-(\rho+p) \gamma^{2} a_{i}-p_{, i}+\frac{1}{4 \pi} \nabla \cdot(\mathbf{V} \times \mathbf{B})(\mathbf{V} \times \mathbf{B})_{i} \\
& -\frac{(\alpha \mathbf{B})_{, i}^{2}}{8 \pi \alpha^{2}}+\frac{\left(\alpha B_{i}\right)_{, j} B^{j}}{4 \pi \alpha}-\frac{1}{4 \pi \alpha}[\mathbf{B} \times\{\mathbf{V} \times(\nabla \times(\alpha \mathbf{V} \times \mathbf{B}))\}]_{i}, \\
& \gamma^{2}\left(\frac{1}{\alpha} \frac{\partial}{\partial t}+\mathbf{V} \cdot \nabla\right)(\rho+p)+2(\rho+p) \gamma^{4} \mathbf{V} \cdot\left(\frac{1}{\alpha} \frac{\partial}{\partial t}+\mathbf{V} \cdot \nabla\right) \mathbf{V}+2(\rho+p) \gamma^{2} \mathbf{V} \cdot \mathbf{a}-\frac{1}{\alpha} \frac{\partial p}{\partial t} \\
& +(\rho+p) \gamma^{2} \nabla \cdot \mathbf{V}+\frac{1}{4 \pi \alpha}\left[(\mathbf{V} \times \mathbf{B}) \cdot(\nabla \times(\alpha \mathbf{B}))+(\mathbf{V} \times \mathbf{B}) \cdot \frac{\partial}{\partial t}(\mathbf{V} \times \mathbf{B})\right]=0 .
\end{aligned}
$$

Eqs.(2.11)-(2.15) are the perfect GRMHD equations for isothermal plasma in the vicinity of the Schwarzschild

black hole. In the rest of the paper, the Fourier analysis method will be applied to the perturbed form of these equations (including the restrictions of backgrounds) to obtain the dispersion relations.

\section{Non-Rotating Background}

In non-rotating background, we apply the Fourier analysis method to the perturbed GRMHD equations given in Appendix A. These equations will lead to the dispersion relations. We obtain solution of dispersion relations as graphs. The same procedure will be applied to rotating background.

We assume the plane wave type solutions of the form $e^{-i(\omega t-k z)}$ for perturbations (Appendix A). Thus the perturbed variables can be expressed as follows

$$
\tilde{\rho}(t, z)=c_{1} e^{-\iota(\omega t-k z)}, \quad \tilde{p}(t, z)=c_{2} e^{-\iota(\omega t-k z)}, \quad v_{z}(t, z)=c_{3} e^{-\iota(\omega t-k z)}, \quad b_{z}(t, z)=c_{4} e^{-\iota(\omega t-k z)},
$$

where $k$ is the wave number and $\omega$ is the angular frequency.

Using Eq.(3.1) the Fourier analyzed form of the perturbed Eqs.(A7)-(A11) specified for the non-rotating background, it follows that

$$
\begin{aligned}
& -\frac{\iota \omega}{\alpha} c_{4}=0, \\
& \iota k c_{4}=0, \\
& c_{1}\left\{-\rho \iota \omega+\iota k \rho \alpha u-(u \alpha p)^{\prime}-\alpha u^{2} \gamma^{2} p u^{\prime}\right\}+c_{2}\left\{-p \iota \omega+\iota k p \alpha u+(u \alpha p)^{\prime}+\alpha u^{2} \gamma^{2} p u^{\prime}\right\} \\
& +c_{3}(\rho+p)\left\{\alpha\left(1+\gamma^{2} u^{2}\right) \iota k-\alpha\left(1-2 \gamma^{2} u^{2}\right)\left(1+\gamma^{2} u^{2}\right) \frac{u^{\prime}}{u}-i \omega \gamma^{2} u\right\}=0, \\
& c_{1} \rho \gamma^{2}\left\{a_{z}+u u^{\prime}\left(1+\gamma^{2} u^{2}\right)\right\}+c_{2}\left\{p \gamma^{2}\left\{a_{z}+u u^{\prime}\left(1+\gamma^{2} u^{2}\right)\right\}+\iota k p+p^{\prime}\right\} \\
& +c_{3}(\rho+p) \gamma^{2}\left[\left(1+\gamma^{2} u^{2}\right)\left(\frac{-\iota \omega}{\alpha}+\iota u k\right)+\left\{u^{\prime}\left(1+\gamma^{2} u^{2}\right)\left(1+4 \gamma^{2} u^{2}\right)+2 \gamma^{2} u a_{z}\right\}\right]=0, \\
& \left\{\rho \gamma^{2}\left(\frac{-\iota \omega}{\alpha}+\iota k u\right)+\gamma^{2} u \rho^{\prime}+\rho 2 \gamma^{2} u a_{z}+\rho\left(1+2 \gamma^{2} u^{2}\right) \gamma^{2} u^{\prime}\right\} c_{1} \\
& +\left\{\frac{-\iota \omega}{\alpha} p\left(\gamma^{2}-1\right)+\iota k \gamma^{2} u p+\gamma^{2} u p^{\prime}+2 \gamma^{2} u p a_{z}+p\left(1+2 \gamma^{2} u^{2}\right) \gamma^{2} u^{\prime}\right\} c_{2} \\
& +(\rho+p) \gamma^{2}\left\{\frac{-2 \iota \omega}{\alpha} \gamma^{2} u+\left(\iota k+3 \gamma^{2} u u^{\prime}+a_{z}\right)\left(1+2 \gamma^{2} u^{2}\right)-\frac{u^{\prime}}{u}\right\} c_{3}=0 .
\end{aligned}
$$

Eqs.(3.2) and (3.3) yield that $c_{4}=0$, i.e., the magnetic field has no effect of black hole gravity as well as time. We would like to mention here that Eqs.(3.4)-(3.6) also give non-magnetized plasma. 


\section{Numerical Solutions}

We consider that the magnetosphere is filled with stiff fluid for which $\rho=$ constant $=p$. We also assume that the time lapse $\alpha=z$. Using these values, the mass conservation law in 3-dimensions, i.e., $\alpha(\rho+p) \gamma u=$ constant gives $u=\frac{1}{\sqrt{1+z^{2}}}$. Due to the magnetic field parallel to the wave number and the use of the longitudinal part of momentum equation, the isothermal plasma shows only the longitudinal (electron or ion) plasma waves.

When we use these values, the determinant of the coefficients of constants $c_{1}, c_{2}$ and $c_{3}$ in Eqs.(3.4)(3.6) leads to a complex dispersion equation. On comparing the real and imaginary parts we obtain two dispersion equations. The real part shows an equation of the type $A_{1}(z) k^{2}+A_{2}(z, \omega) k+A_{3}(z, \omega)=0$ which on solving gives two values of the wave number $k$. The imaginary part gives the dispersion equation of the type $A_{1}(z) k^{3}+A_{2}(z, \omega) k^{2}+A_{3}(z, \omega) k+A_{4}(z, \omega)=0$ which provides three (including two complex conjugate) values of the wave number. Using the values of $k$, the quantities like phase velocity $\left(v_{p}=\frac{\omega}{k}\right)$, refractive index $\left(n=\frac{1}{v_{p}}\right)$, its change with respect to angular frequency $\left(\frac{d n}{d \omega}\right)$ and group velocity $\left(v_{g}=\frac{d \omega}{d k}=\frac{1}{n+\omega \frac{d n}{d \omega}}\right)$ can be obtained. These quantities help us to investigate the properties of the waves as well as their dispersion.

The two dispersion relations obtained from the real part give the same value of $k$. The corresponding graphs are given in Figure 1. The wave number obtained from the imaginary part is shown in Figure 2.

In Figure 1, the wave number $k$ becomes infinite at $z=0$ which means that the waves vanish at the horizon due to effect of immense gravity. No wave with zero angular frequency exist which is a physical consequence. The wave number decreases as we go away from the horizon which means that the waves lose energy as their distance from the horizon increases. The wave number increases with the increase in angular frequency. The phase and group velocities are positive and increase when we go away from the horizon. We can deduce that the increase in gravity decreases the wave velocity as well as the speed with which energy travels. Both the phase and group velocities are equal. The refractive index is greater than one and there is no change with respect to angular frequency hence the dispersion is not normal there [32].

Figure 2 shows that the wave number is infinite at $z=0$. In the region $0<z<1,0 \leq \omega<6 \times 10^{-6}$, the wave number takes some complex value and the waves are evanescent there, otherwise the wave number $k$ is positive. It decreases as we move away from the event horizon of the Schwarzschild black hole. The increase in angular frequency increases the wave number. The phase and group velocities are equal except in the region $0<\omega<6 \times 10^{-6}$. Since the phase velocity increases as we go away from the horizon, the waves gain energy as they go far away from the event horizon of the black hole. Refractive index is positive and greater than one but its derivative with respect to angular frequency has random variation. Hence the medium is not of normal dispersion.

\section{Rotating Non-Magnetized Background}

The perturbed form of GRMHD Eqs.(2.11)-(2.15) meant for rotating non-magnetized background are given in Appendix B. For the sake of Fourier analysis, we assume that the perturbations (Appendix B) are of the plane wave form, i.e., $e^{-i(\omega t-k z)}$. Thus the perturbed variables take the form

$$
\tilde{\rho}(t, z)=c_{1} e^{-\iota(\omega t-k z)}, \quad \tilde{p}(t, z)=c_{2} e^{-\iota(\omega t-k z)}, \quad v_{z}(t, z)=c_{3} e^{-\iota(\omega t-k z)}, \quad v_{x}(t, z)=c_{4} e^{-\iota(\omega t-k z)} .
$$

Using Eq.(4.1), we can write the Fourier analyzed Eqs.(B7)-(B10) for rotating non-magnetized background 

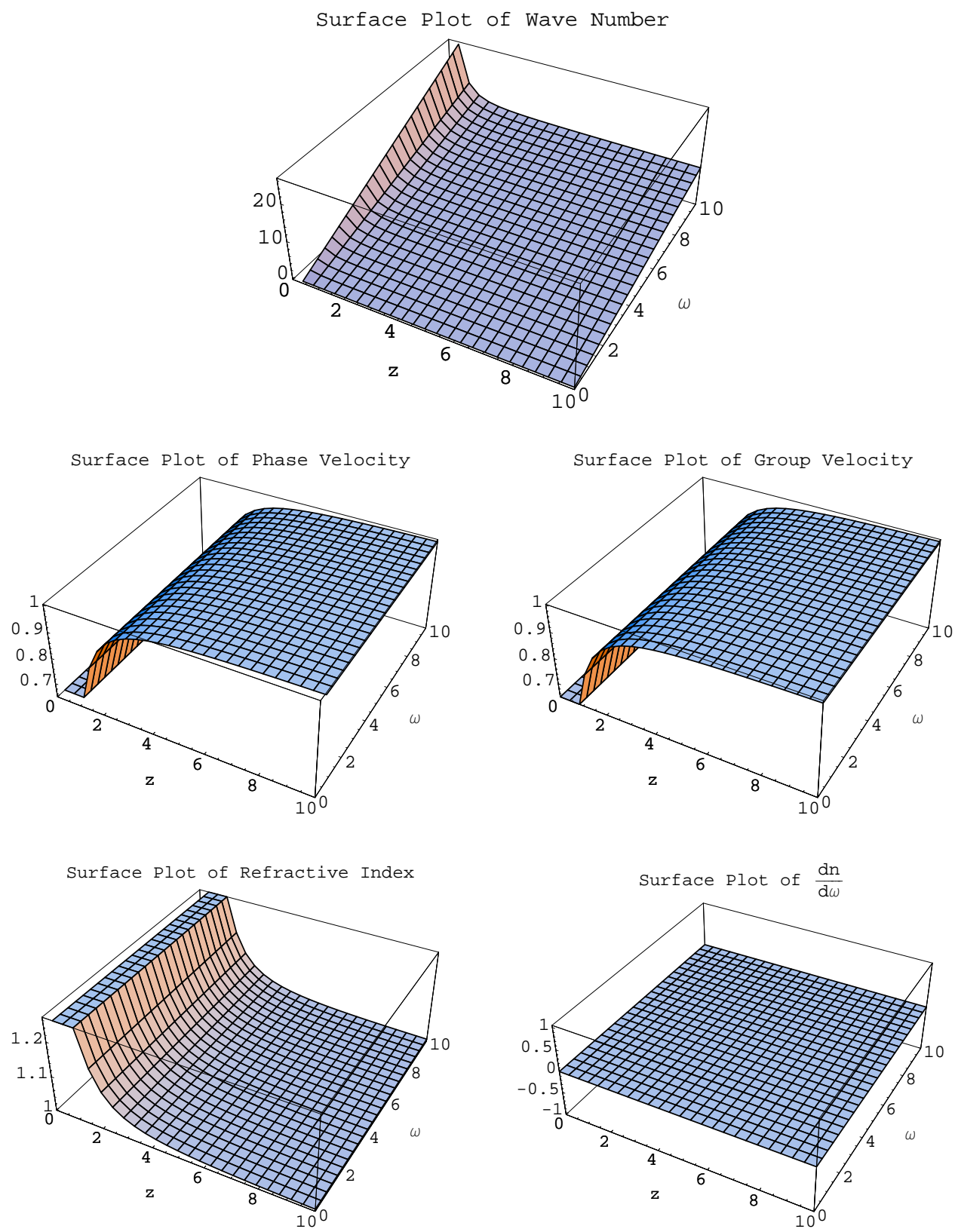

Figure 1: The waves decrease as they move away from the horizon. The phase and group velocities are equal. The dispersion is not normal. 

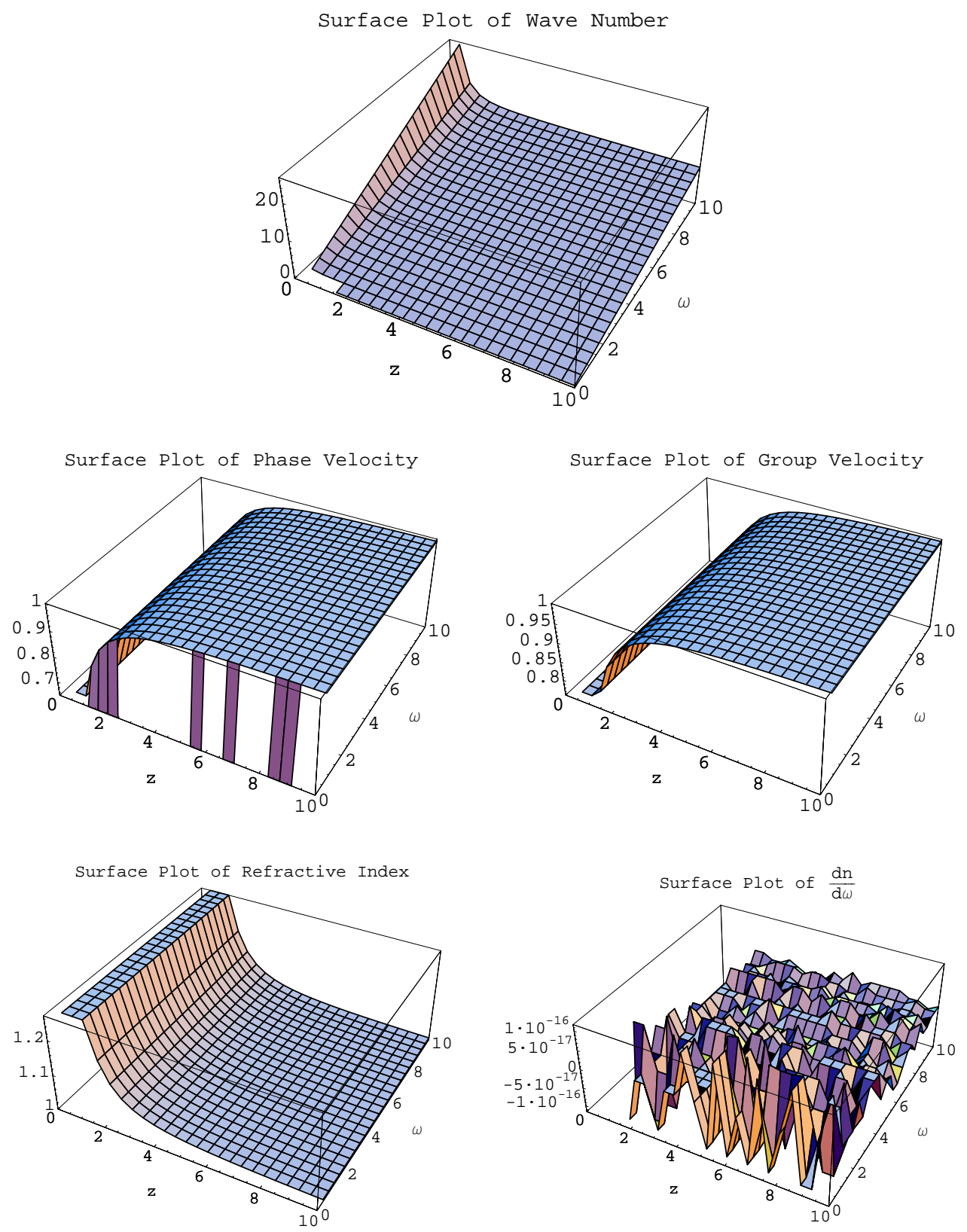

Figure 2: The wave number decreases as the waves move away from the event horizon. In most of the region the phase and group velocities are equal and the dispersion is not normal. 
as follows

$$
\begin{aligned}
& c_{1}\left\{(-\iota \omega+\iota \alpha k u) \rho-\alpha^{\prime} u p-\alpha u^{\prime} p-\alpha u p^{\prime}-\alpha \gamma^{2} u p\left(V V^{\prime}+u u^{\prime}\right)\right\} \\
& +c_{2}\left\{(-\iota \omega+\iota \alpha k u) p+\alpha^{\prime} u p+\alpha u^{\prime} p+\alpha u p^{\prime}+\alpha \gamma^{2} u p\left(V V^{\prime}+u u^{\prime}\right)\right\} \\
& +c_{3}(\rho+p)\left[-\iota \omega \gamma^{2} u+\iota k \alpha\left(1+\gamma^{2} u^{2}\right)-\alpha\left\{\left(1-2 \gamma^{2} u^{2}\right)\left(1+\gamma^{2} u^{2}\right) \frac{u^{\prime}}{u}-2 \gamma^{4} u^{2} V V^{\prime}\right\}\right] \\
& +c_{4}(\rho+p)\left[\gamma^{2} V(-\iota \omega+\iota k \alpha u)+\alpha \gamma^{2} u\left\{\left(1+2 \gamma^{2} V^{2}\right) V^{\prime}+2 \gamma^{2} u V u^{\prime}\right\}\right]=0, \\
& c_{1} \rho \gamma^{2} u\left\{\left(1+\gamma^{2} V^{2}\right) V^{\prime}+\gamma^{2} u V u^{\prime}\right\}+c_{2} p \gamma^{2} u\left\{\left(1+\gamma^{2} V^{2}\right) V^{\prime}+\gamma^{2} u V u^{\prime}\right\} \\
& c_{3}(\rho+p) \gamma^{2}\left[\gamma^{2} u V\left(\frac{-\iota \omega}{\alpha}+\iota k u\right)+\left\{\left(1+2 \gamma^{2} V^{2}\right)\left(1+2 \gamma^{2} u^{2}\right)-\gamma^{2} V^{2}\right\} V^{\prime}+2 \gamma^{2} u V u^{\prime}\left(1+2 \gamma^{2} u^{2}\right)\right] \\
& +c_{4}(\rho+p) \gamma^{2}\left[\left(1+\gamma^{2} V^{2}\right)\left(\frac{-\iota \omega}{\alpha}+\iota k u\right)+\gamma^{2} u\left\{\left(1+4 \gamma^{2} u^{2}\right) u u^{\prime}+4\left(1+\gamma^{2} V^{2}\right) V V^{\prime}\right\}\right]=0 \text {, } \\
& c_{1} \rho \gamma^{2}\left\{a_{z}+u u^{\prime}\left(1+\gamma^{2} u^{2}\right)+\gamma^{2} u^{2} V V^{\prime}\right\}+c_{2}\left[p \gamma^{2}\left\{a_{z}+u u^{\prime}\left(1+\gamma^{2} u^{2}\right)+\gamma^{2} u^{2} V V^{\prime}\right\}+p^{\prime}+\iota k p\right] \\
& c_{3}(\rho+p) \gamma^{2}\left[\left(1+\gamma^{2} u^{2}\right)\left(\frac{-\iota \omega}{\alpha}+\iota k u\right)+u^{\prime}\left(1+\gamma^{2} u^{2}\right)\left(1+4 \gamma^{2} u^{2}\right)+2 u \gamma^{2}\left\{\left(1+2 \gamma^{2} u^{2}\right) V V^{\prime}+a_{z}\right\}\right] \\
& +c_{4}(\rho+p) \gamma^{4}\left[u V\left(\frac{-\iota \omega}{\alpha}+\iota k u\right)+u^{2} V^{\prime}\left(1+4 \gamma^{2} u^{2}\right)+2 V\left\{\left(1+2 \gamma^{2} u^{2}\right) u u^{\prime}+a_{z}\right\}\right]=0, \\
& c_{1}\left[\rho \gamma^{2}\left(\frac{-\iota \omega}{\alpha}+\iota k u\right)+2 \rho \gamma^{2} u\left\{a_{z}+\gamma^{2}\left(V V^{\prime}+u u^{\prime}\right)\right\}+\rho \gamma^{2} u^{\prime}+\rho^{\prime} \gamma^{2} u\right]+c_{2}\left[p\left\{\frac{-\iota \omega}{\alpha}\left(\gamma^{2}-1\right)+\iota k \gamma^{2} u\right\}\right. \\
& \left.+2 p \gamma^{2} u\left\{a_{z}+\gamma^{2}\left(V V^{\prime}+u u^{\prime}\right)\right\}+p \gamma^{2} u^{\prime}+p^{\prime} \gamma^{2} u\right]+c_{3}(\rho+p)\left\{2 \gamma^{4} u \frac{-\iota \omega}{\alpha}+\gamma^{2} \iota k\left(1+2 \gamma^{2} u^{2}\right)\right. \\
& \left.-\gamma^{2} \frac{u^{\prime}}{u}+6 \gamma^{6} u^{2}\left(V V^{\prime}+u u^{\prime}\right)+\gamma^{4}\left(V V^{\prime}+u u^{\prime}\right)+2 \gamma^{4} u u^{\prime}+\gamma^{2} a_{z}\left(1+2 \gamma^{2} u^{2}\right)\right\} \\
& \left.+c_{4}(\rho+p)\left[2 \gamma^{4} V\left(\frac{-\iota \omega}{\alpha}+\iota k u\right)+2 \gamma^{4}\left\{3 \gamma^{2} u V\left(V V^{\prime}+u u^{\prime}\right)+u V^{\prime}+u V a_{z}\right)\right\}\right]=0
\end{aligned}
$$

\section{Numerical Solutions}

We consider time lapse $\alpha=z$ and assume that $V=u$ in the stiff fluid of constant density. Using mass conservation law in three dimensions (with constant mass density), we obtain the value of $u=\frac{1}{\sqrt{2+z^{2}}}$. Since the magnetic field is absent, the plasma contains the longitudinal electron plasma waves, ion plasma waves or the transverse electromagnetic waves.

The determinant of the coefficients of constants $c_{1}, c_{2}, c_{3}$ and $c_{4}$ from Eqs. (4.2)-(4.5) gives a complex dispersion equation. The real part leads to dispersion equation of the type $A_{1}(z) k^{4}+A_{2}(z, \omega) k^{3}+A_{3}(z, \omega) k^{2}+$ $A_{4}(z, \omega) k+A_{5}(z, \omega)=0$. From the imaginary part, we obtain equation of the type $A_{1}(z) k^{3}+A_{2}(z, \omega) k^{2}+$ $A_{3}(z, \omega) k+A_{4}(z, \omega)=0$. The dispersion relation from the real part gives four values of $k$ out of which two are interesting. The other two values turn out to be imaginary in the whole region. The imaginary part of the determinant gives three values out of which two are complex conjugates. The wave numbers obtained from the real part are shown in Figures 3 and 4 while Figure 5 demonstrates the value of $k$ obtained from the imaginary part.

We see from Figure 3 that $k$ is positive for $0<z \leq 10,0.011<\omega \leq 10$ while it is negative for $0<z \leq$ $1,0<\omega \leq 0.011$. It is interesting to note that in this small region, the phase velocity and refractive index are negative whereas the group velocity is positive. The fluid shows the properties of Veselago medium 33 . The wave number decreases with the increase in $z$ and increases with the increase in angular frequency in the region $1<z \leq 10,0.011<\omega \leq 10$. In the neighborhood of $\omega=0$, the phase velocity is large. It decreases with the increase in $z$ with the exception of the interval where it has complex values. The group velocity shows the similar behavior with the exception that it is complex at $\omega=0$. In the region $0<z \leq 2,2<\omega \leq 10$, the index of refraction is greater than one and also $\frac{d n}{d \omega}>0$. The dispersion is therefore normal in this small region but not normal otherwise.

In Figure 4, the wave number becomes imaginary in the region $0 \leq \omega \leq 0.5$ and hence evanescent waves exist there. The wave number increases with an increase in $\omega$ and the waves lose energy as we go away from 

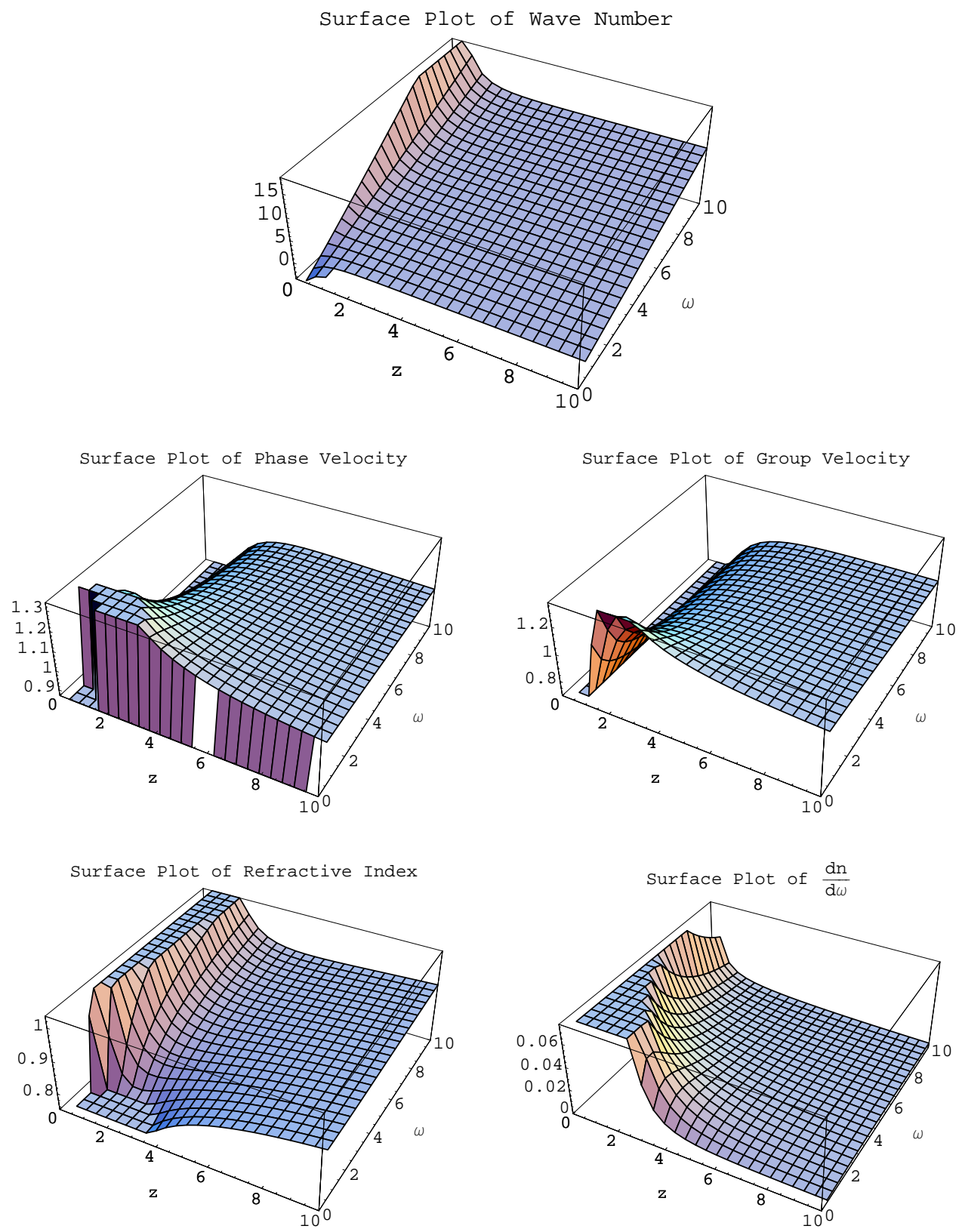

Figure 3: The wave number decreases when waves move away from the event horizon. A small region is showing properties of Veselago medium. The dispersion is not normal in most of the region. 

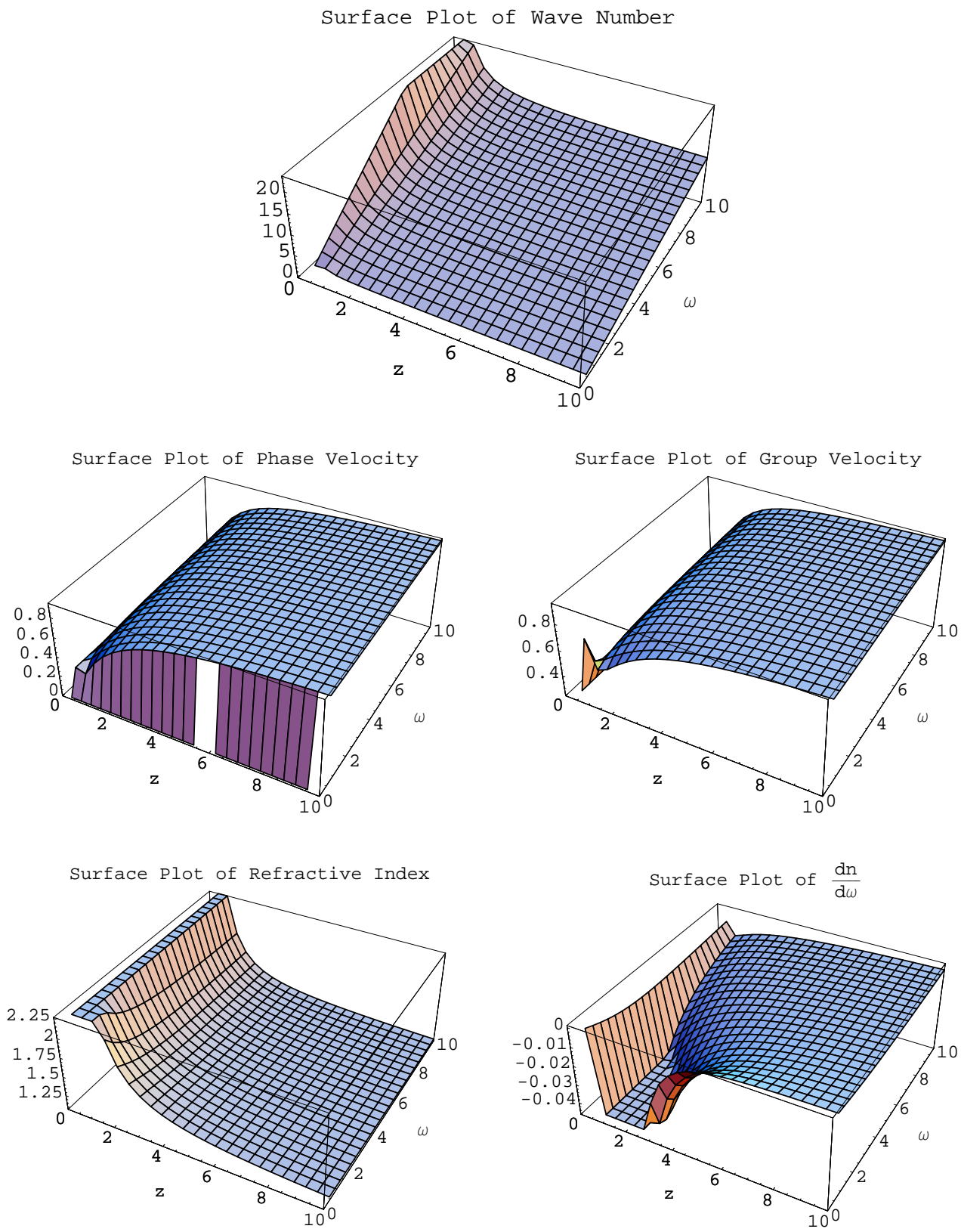

Figure 4: The wave number decreases as the waves move away from the event horizon. The dispersion of waves is found to be not normal. 
the horizon. In the region $0<z \leq 10,0.5<\omega \leq 10$, the wave number as well as the phase and group velocities are real. The refractive index is greater near the horizon due to immense gravitational field and decreases away from the horizon. The change of refractive index with respect to the angular velocity is negative in the whole region. Thus the dispersion is not normal.

Figure 5 gives the description of the wave number obtained from the imaginary part of the determinant. The wave number increases as they move towards the horizon of the black hole. It also increases as the angular frequency of waves increases. The waves are evanescent in the region $0 \leq z<0.112$ while the real waves lie in the region $0.112 \leq z \leq 10$. The phase velocity is zero at $z=0.112$ and it increases towards 1 as $z$ increases. The refractive index is greater than one in this region but its change with respect to angular frequency is negative which shows that dispersion is not normal.

\section{$5 \quad$ Rotating Magnetized Background}

This is the most general case. The perturbed GRMHD equations for this situation are given in Appendix C. Since these equations include the additional magnetic field, therefore the Fourier analysis procedure demands that the magnetic field perturbations should have harmonic space and time dependence, i.e., of the form $\mathbf{b} \sim e^{-\iota(\omega t-k z)}$. These perturbed variables admit the following notations

$$
b_{z}(t, z)=c_{5} e^{-\iota(\omega t-k z)}, \quad b_{x}(t, z)=c_{6} e^{-\iota(\omega t-k z)} .
$$

When we substitute these values in component form of perturbed Eqs.(C2) and (C3) (Appendix C), it follows that

$$
c_{5}\left(-\frac{\iota \omega}{\alpha}+\iota k u\right)=0, \quad \iota k c_{5}=0 .
$$

Both these equations yield that $c_{5}=0$ and hence $b_{z}=0$. Substituting this value in addition to the values from 

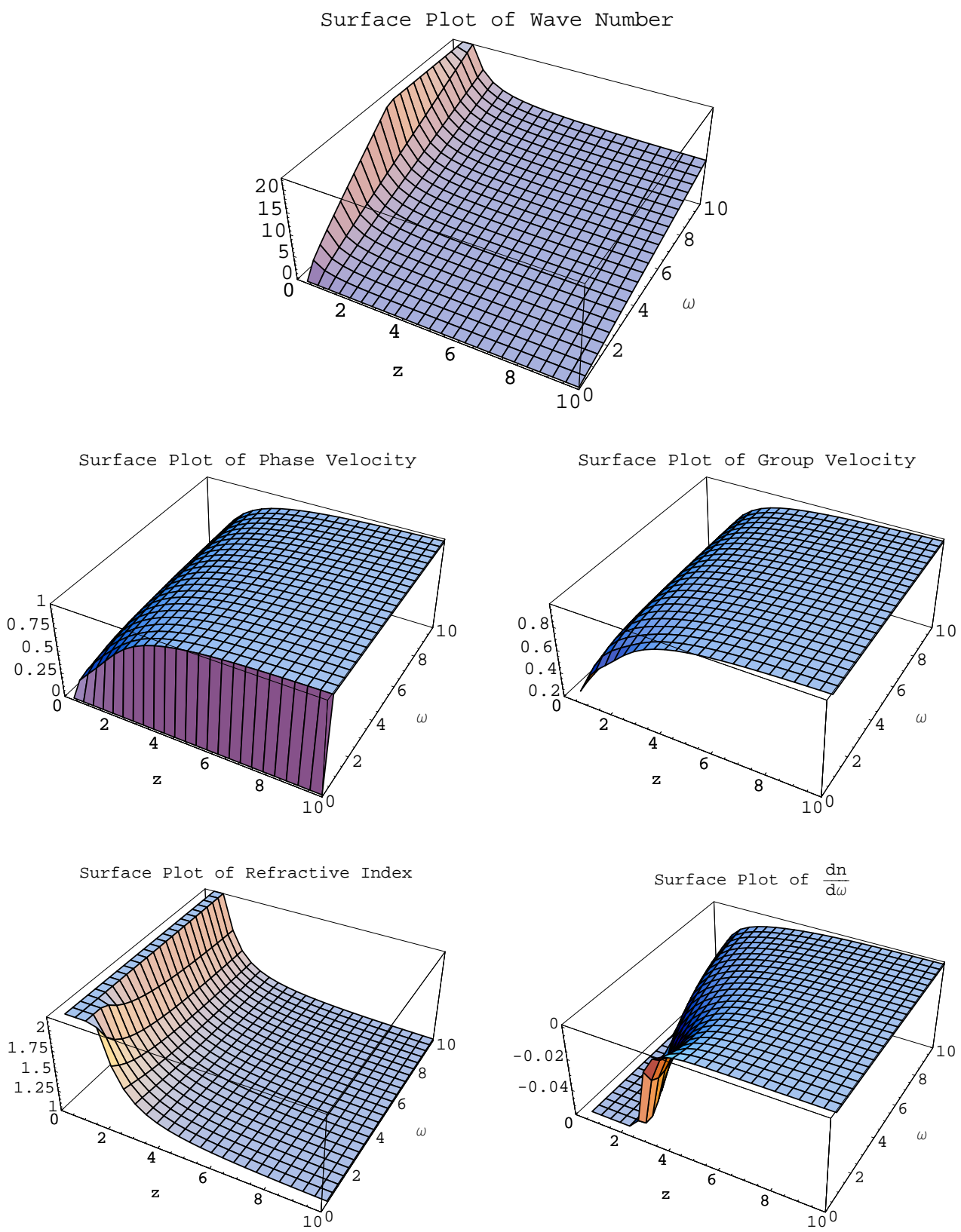

Figure 5: The wave number decreases as the waves move away from the horizon of the black hole. The waves are not normally dispersed. 
Eqs.(4.1) and (5.1) in Eqs.(C1), (C4)-(C7), we obtain the following Fourier analyzed equations

$$
\begin{aligned}
& -c_{3}\left\{(\alpha \lambda)^{\prime}+\iota k \alpha \lambda\right\}+c_{4}\left(\alpha^{\prime}+\iota k \alpha\right)-c_{6}\left\{(\alpha u)^{\prime}-\iota \omega+\iota k \alpha u\right\}=0, \\
& c_{1}\left\{\rho(-\iota \omega+\iota k \alpha u)-\alpha^{\prime} u p-\alpha u^{\prime} p-\alpha u p^{\prime}-\alpha u p \gamma^{2}\left(V V^{\prime}+u u^{\prime}\right)\right\} \\
& +c_{2}\left\{p(-\iota \omega+\iota k \alpha u)+\alpha^{\prime} u p+\alpha u^{\prime} p+\alpha u p^{\prime}+\alpha u p \gamma^{2}\left(V V^{\prime}+u u^{\prime}\right)\right\} \\
& +c_{3}(\rho+p)\left[-\iota \omega \gamma^{2} u+\iota k \alpha\left(1+\gamma^{2} u^{2}\right)-\alpha\left\{\left(1-2 \gamma^{2} u^{2}\right)\left(1+\gamma^{2} u^{2}\right) \frac{u^{\prime}}{u}-2 \gamma^{4} u^{2} V V^{\prime}\right\}\right] \\
& +c_{4}(\rho+p) \gamma^{2}\left[(-\iota \omega+\iota k \alpha u) V+\alpha u\left\{\left(1+2 \gamma^{2} V^{2}\right) V^{\prime}+2 \gamma^{2} u V u^{\prime}\right\}\right]=0, \\
& c_{1} \rho \gamma^{2} u\left\{\left(1+\gamma^{2} V^{2}\right) V^{\prime}+\gamma^{2} u V u^{\prime}\right\}+c_{2} p \gamma^{2} u\left\{\left(1+\gamma^{2} V^{2}\right) V^{\prime}+\gamma^{2} u V u^{\prime}\right\} \\
& +c_{3}\left[-\left\{(\rho+p) \gamma^{4} u V-\frac{\lambda B^{2}}{4 \pi}\right\} \frac{\iota \omega}{\alpha}+\iota k u\left\{(\rho+p) \gamma^{4} u V+\frac{\lambda B^{2}}{4 \pi}\right\}\right. \\
& \left.+(\rho+p) \gamma^{2}\left\{\left\{\left(1+2 \gamma^{2} u^{2}\right)\left(1+2 \gamma^{2} V^{2}\right)-\gamma^{2} V^{2}\right\} V^{\prime}+2 \gamma^{2}\left(1+2 \gamma^{2} u^{2}\right) u V u^{\prime}\right\}+\frac{B^{2} u}{4 \pi \alpha}(\lambda \alpha)^{\prime}\right] \\
& +c_{4}\left[-\left\{(\rho+p) \gamma^{2}\left(1+\gamma^{2} V^{2}\right)+\frac{B^{2}}{4 \pi}\right\} \frac{\iota \omega}{\alpha}+\iota k u\left\{(\rho+p) \gamma^{2}\left(1+\gamma^{2} V^{2}\right)-\frac{B^{2}}{4 \pi}\right\}\right. \\
& \left.+(\rho+p) \gamma^{4} u\left\{\left(1+4 \gamma^{2} V^{2}\right) u u^{\prime}+4\left(1+\gamma^{2} V^{2}\right) V V^{\prime}\right\}-\frac{B^{2} u \alpha^{\prime}}{4 \pi \alpha}\right] \\
& -\frac{B^{2}}{4 \pi} c_{6}\left\{\left(1-u^{2}\right) \iota k+\frac{\alpha^{\prime}}{\alpha}\left(1-u^{2}\right)-u u^{\prime}\right\}=0 \text {, } \\
& c_{1} \gamma^{2} \rho\left[a_{z}+u\left\{\left(1+\gamma^{2} u^{2}\right) u^{\prime}+\gamma^{2} V u V^{\prime}\right\}\right]+c_{2}\left[\gamma^{2} p\left\{a_{z}+u\left\{\left(1+\gamma^{2} u^{2}\right) u^{\prime}+\gamma^{2} V u V^{\prime}\right\}\right\}+\iota k p+p^{\prime}\right] \\
& +c_{3}\left[-\left\{(\rho+p) \gamma^{2}\left(1+\gamma^{2} u^{2}\right)+\frac{\lambda^{2} B^{2}}{4 \pi}\right\} \frac{\iota \omega}{\alpha}+\left\{(\rho+p) \gamma^{2}\left(1+\gamma^{2} u^{2}\right)-\frac{\lambda^{2} B^{2}}{4 \pi}\right\} \iota k u\right. \\
& \left.+\left\{(\rho+p) \gamma^{2}\left\{u^{\prime}\left(1+\gamma^{2} u^{2}\right)\left(1+4 \gamma^{2} u^{2}\right)+2 u \gamma^{2}\left\{\left(1+2 \gamma^{2} u^{2}\right) V V^{\prime}+a_{z}\right\}\right\}-\frac{\lambda B^{2} u}{4 \pi \alpha}(\alpha \lambda)^{\prime}\right\}\right] \\
& +c_{4}\left[-\left\{(\rho+p) \gamma^{4} u V-\frac{\lambda B^{2}}{4 \pi}\right\} \frac{\iota \omega}{\alpha}+\left\{(\rho+p) \gamma^{4} u V+\frac{\lambda B^{2}}{4 \pi}\right\} \iota k u\right. \\
& \left.+\left\{(\rho+p) \gamma^{4}\left\{u^{2} V^{\prime}\left(1+4 \gamma^{2} V^{2}\right)+2 V\left\{a_{z}+u u^{\prime}\left(1+2 \gamma^{2} u^{2}\right)\right\}\right\}+\frac{\lambda B^{2} \alpha^{\prime} u}{4 \pi \alpha}\right\}\right] \\
& +\frac{B^{2}}{4 \pi} c_{6}\left\{\lambda\left(1-u^{2}\right) \iota k+\lambda \frac{\alpha^{\prime}}{\alpha}\left(1-u^{2}\right)-\lambda u u^{\prime}+\frac{(\lambda \alpha)^{\prime}}{\alpha}\right\}=0, \\
& c_{1} \gamma^{2}\left[\rho\left(\frac{-\iota \omega}{\alpha}+\iota k u\right)+2 \rho u\left\{a_{z}+\gamma^{2}\left(V V^{\prime}+u u^{\prime}\right)\right\}+\rho u^{\prime}+\rho^{\prime} u\right]+c_{2}\left[p\left\{\frac{-\iota \omega}{\alpha}\left(\gamma^{2}-1\right)+\iota k \gamma^{2} u\right\}\right. \\
& \left.+2 p \gamma^{2} u\left\{a_{z}+\gamma^{2}\left(V V^{\prime}+u u^{\prime}\right)\right\}+p \gamma^{2} u^{\prime}+p^{\prime} \gamma^{2} u\right]+c_{3}\left[( \rho + p ) \left\{2 \gamma^{4} u \frac{-\iota \omega}{\alpha}+\gamma^{2} \iota k\left(1+2 \gamma^{2} u^{2}\right)\right.\right. \\
& \left.-\gamma^{2} \frac{u^{\prime}}{u}+6 \gamma^{6} u^{2}\left(V V^{\prime}+u u^{\prime}\right)+\gamma^{4}\left(V V^{\prime}+u u^{\prime}\right)+2 \gamma^{4} u u^{\prime}+\gamma^{2} a_{z}\left(1+2 \gamma^{2} u^{2}\right)\right\} \\
& +\frac{B^{2}}{4 \pi \alpha}\left\{\lambda(\alpha \lambda)^{\prime}-u(\alpha \lambda)^{\prime}(u \lambda-V)-\iota(k \alpha u+\omega) \lambda(u \lambda-V)\right]+c_{4}\left[2 \gamma ^ { 4 } ( \rho + p ) \left\{V\left(\frac{-\iota \omega}{\alpha}+\iota k u\right)\right.\right. \\
& \left.\left.\left.+\left\{3 \gamma^{2} u V\left(V V^{\prime}+u u^{\prime}\right)+u V^{\prime}+u V a_{z}\right)\right\}\right\}+\frac{B^{2}}{4 \pi \alpha}\left\{-(\alpha \lambda)^{\prime}+\alpha^{\prime} u(u \lambda-V)+(\iota k \alpha u+\iota \omega)(u \lambda-V)\right\}\right] \\
& +c_{6} \frac{B^{2}}{4 \pi \alpha}\left[u(\alpha \lambda)^{\prime}+\left\{\alpha^{\prime}-u(\alpha u)^{\prime}+\iota k\left(1-u^{2}\right)\right\}(u \lambda-V)\right]=0 .
\end{aligned}
$$

\section{Numerical Solutions}

We take the same assumptions as in the case of rotating non-magnetized plasma, i.e., $u=\frac{1}{\sqrt{z^{2}+2}}=V$. Substituting this value in $V=\frac{1}{\alpha}+\lambda u$ of [21], it turns out that $\lambda=1-\frac{\sqrt{z^{2}+2}}{z}$. This shows that the magnetic field 
diverges near the horizon (as discussed in [6]). Also, $B^{2}$ is taken to be $\frac{176}{7}$.

The matrix of the coefficients of constants $c_{1}, c_{2}, c_{3}, c_{4}$ and $c_{6}$ is of order $5 \times 5$ whose determinant gives a dispersion equation quartic in $k$ from the real part and a quintic from the imaginary part. The real part leads to four interesting solutions whereas the imaginary part is difficult to solve to get exact solutions. It is obvious that the magnetic field is non-zero and the wave number is in arbitrary direction to the magnetic field. Since the external magnetic field is present, the plasma is anisotropic. This magnetic field has profound effects in plasma wave modes discussed in the previous section.

The real part gives dispersion relation of the type $A_{1}(z) k^{4}+A_{2}(z, \omega) k^{3}+A_{3}(z, \omega) k^{2}+A_{4}(z, \omega) k+A_{5}(z, \omega)=0$. It gives four values of $k$ out of which two are complex conjugate roots and are not interesting. The two interesting roots provided by the real part are shown in Figures 6 and 7.

In Figure 6, waves are evanescent in the regions $0<z<1.65$ and $0<\omega<2.5 \times 10^{-4}$. The wave number becomes infinite at zero angular frequency as well as at $z=0$ and hence waves do not exist there. Real waves are present in rest of the region. We see that the wave number is large near the horizon and decreases gradually afterwards as $z$ increases. Also, $k$ increases with an increase in the angular frequency. The phase and group velocities increase as we go away from the horizon. Also, the phase velocity is zero at zero angular frequency but the group velocity is infinite there. The refractive index is less than one in the whole region and hence the dispersion is not normal.

Figure 7 shows that the evanescent waves lie in the regions $0<z \leq 1.65$ and $0 \leq \omega \leq 0.0005$. The wave number is infinite at $z=0$ but decreases with an increase in $z$ and increases with the increase in the angular frequency. The group velocity is greater than the phase velocity which indicates that the dispersion is anomalous. The value of refractive index is less than one which confirms this indication.

The imaginary part of the determinant gives a dispersion relation quintic in $k$, i.e., $A_{1}(z) k^{5}+A_{2}(z, \omega) k^{4}+$ $A_{3}(z, \omega) k^{3}+A_{4}(z, \omega) k^{2}+A_{5}(z, \omega) k+A_{6}(z, \omega)=0$. This equation cannot be solved analytically for the exact solutions and is solved numerically using software Mathematica. The roots are approximated in arrays for each point of the two-dimensional mesh with equal step lengths 0.2 for $z$ and $\omega$. This equation gives one real root and two sets of complex conjugate roots. The real data values for the root gives a real interpolation function. This function represents the value of $k$ shown graphically which is used to investigate the properties of medium.

When we substitute the assumptions, the approximated root becomes infinite at $z=0$ due to which no wave exists there. We omit the value $z=0$ and our mesh reduces to $0.2 \leq z \leq 10,0 \leq \omega \leq 10$ for the interpolating function. The corresponding real root is shown in Figure 8.

Figure 8 shows that the wave number is high near the horizon. It abruptly decreases and gradually increases as we go away from the horizon. The real wave number is shown in the figure. The wave number becomes negative in the region $0.845 \leq z \leq 10$. Also, the phase and group velocities as well as refractive index are negative there which is the property of Veselago medium. The phase velocity is greater than the group velocity in the whole region admitting real waves. Also, the change in refractive index with respect to the angular frequency is greater than zero for the region $1.61 \leq z \leq 10,0<\omega \leq 10$, thus it admits normal dispersion of waves 34. The change in refractive index with respect to the angular frequency is less than zero for the region $0.2 \leq z<1.61,0<\omega \leq 10$ which shows anomalous dispersion of waves.

\section{Conclusion}

We derive the GRMHD equations for the Schwarzschild black hole magnetosphere in $3+1$ formalism. We discuss one dimensional perturbations in perfect MHD condition. This has been explored for isothermal state of plasma. These equations are written in component form and then Fourier analyzed by using the assumption of plane waves. The determinant of the coefficients is solved for the following three cases in the magnetosphere:

1. Non-Rotating Background (either non-magnetized or magnetized);

2. Rotating Non-Magnetized Background;

3. Rotating Magnetized Background.

The non-rotating background is the pure Schwarzschild geometry outside the event horizon. The rotating background is the restricted Kerr geometry in the vicinity of the event horizon which admits a variable lapse function with negligible rotation. The dispersion relations and consequently, the wave numbers are obtained 

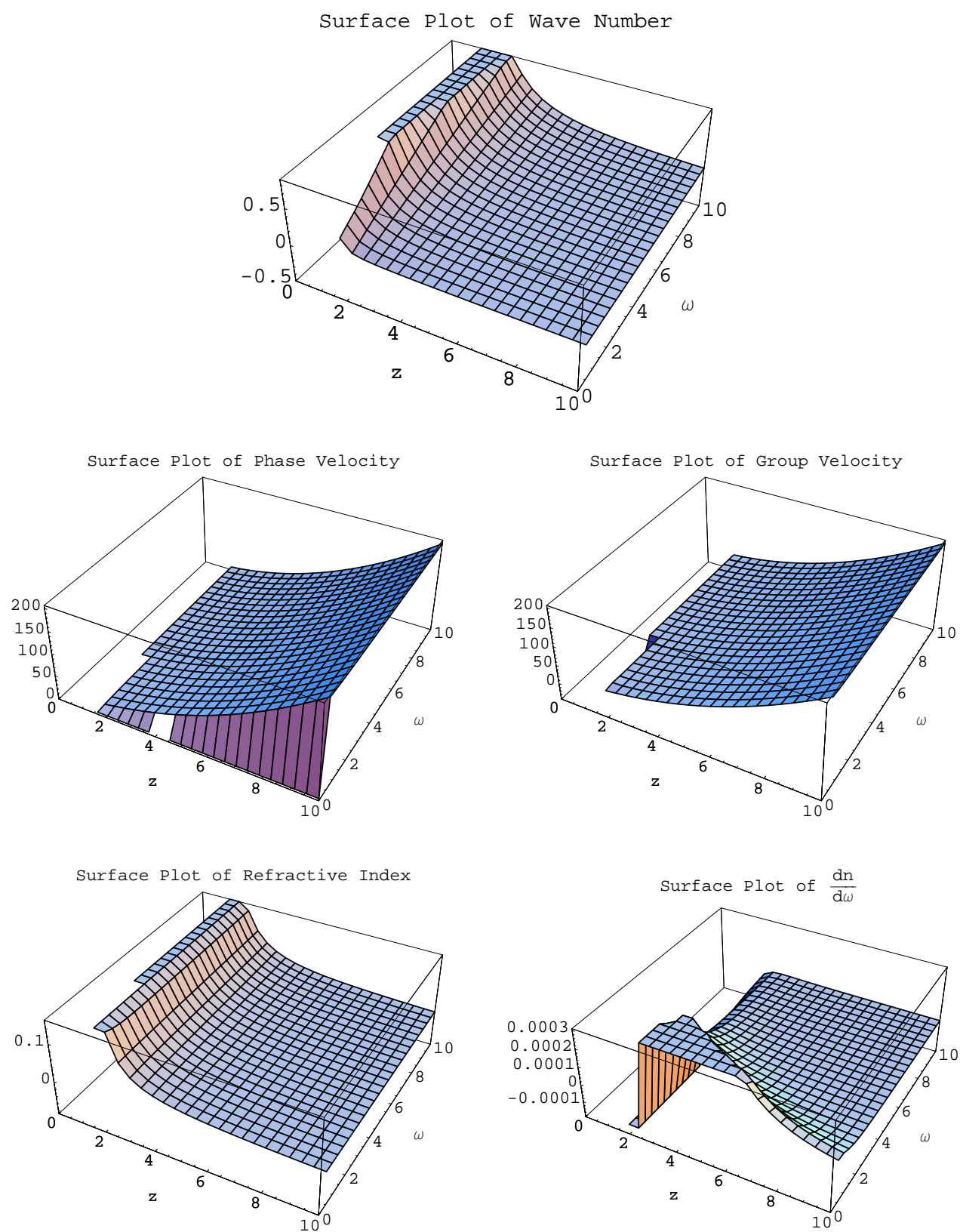

Figure 6: The wave number decreases as the waves move away from the event horizon. The phase and group velocities are equal in most of the region. The dispersion is not normal. 

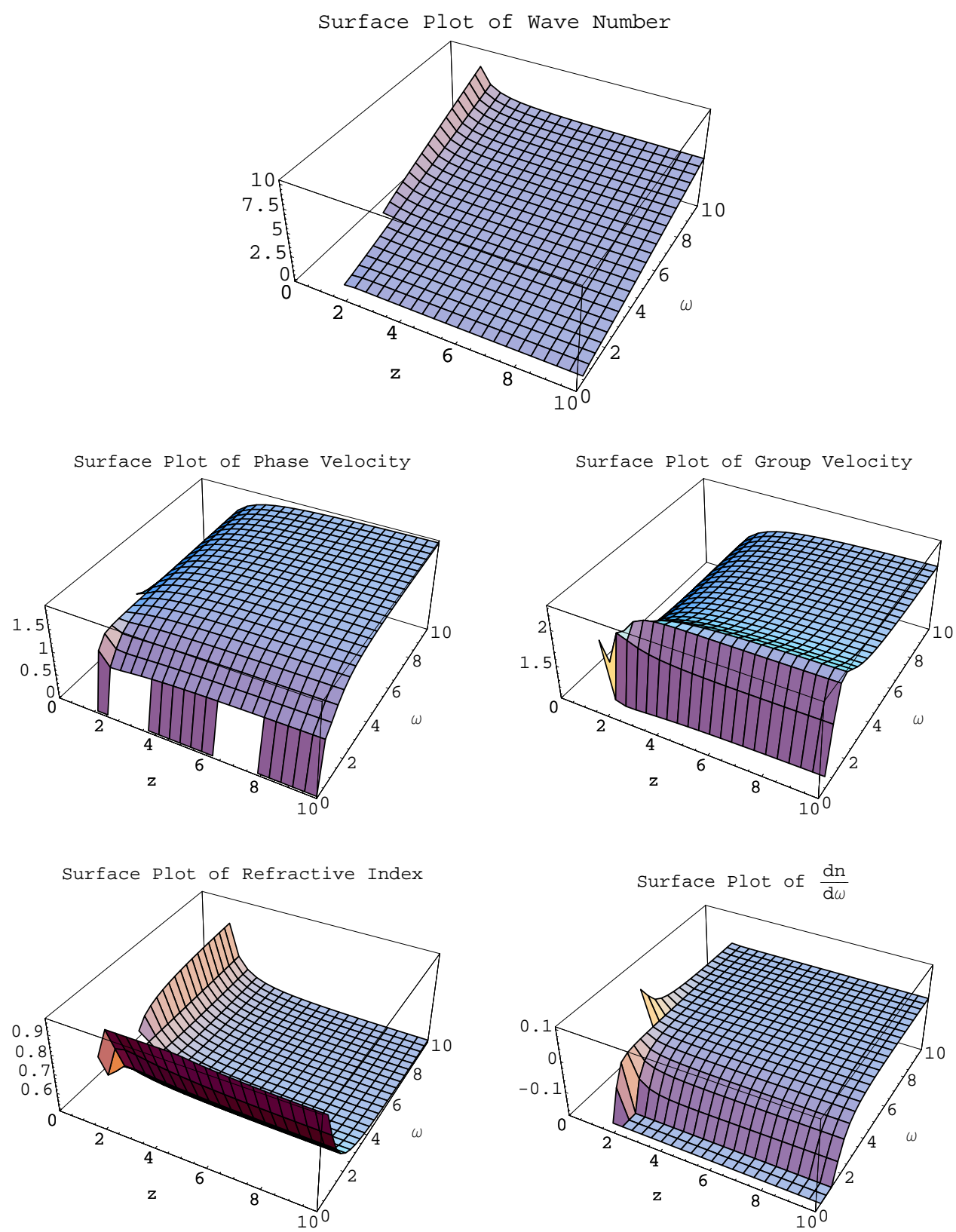

Figure 7: The waves decreases in number as we move away from the event horizon of the black hole. The group velocity is greater than the phase velocity. The dispersion is anomalous in the region. 

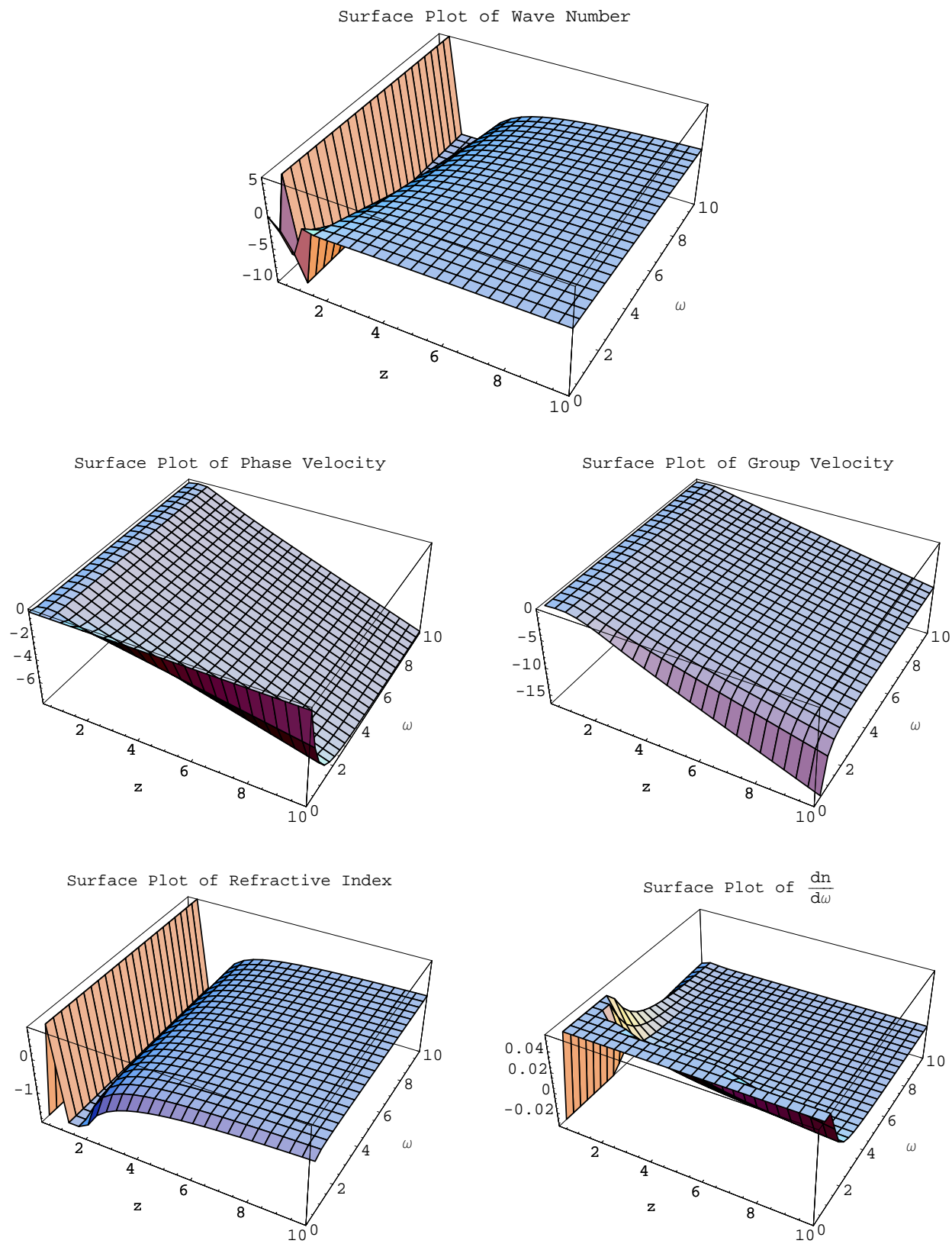

Figure 8: Some of the region possess the properties of Veselago medium. The phase velocity is greater than the group velocity in most of the region for which the dispersion is normal. 
for these backgrounds. The properties of plasma are inferred on the basis of this number and the corresponding quantities are obtained in the form of graphs.

We find that the gravity effect can be observed in the perturbations both in the non-rotating and rotating backgrounds. The values of $k$ only show the gravitational effects on the smooth harmonic wave type perturbations. The rotation of the background adds this effect to the perturbed quantities. It can also be seen that the magnetic field perturbations are influenced in the case of rotating background whereas there is no effect of gravitation on the perturbed magnetic field in the non-rotating background. This implies that rotation affects the magnetic field. It is interesting to note that wave numbers decrease and the phase and group velocities increase as we go away from the horizon in each case.

Recently, we have discussed the properties of the Schwarzschild magnetosphere for the cold plasma case 29 and some interesting consequences have been obtained about the wave analysis. In this paper, we have extended this analysis by adding the pressure effect (isothermal plasma). It turns out that pressure has brought changes in the case of non-rotating, non-magnetized background. In the case of cold plasma, normal dispersion of the waves was found for the non-rotating background whereas in the similar case of isothermal plasma, the dispersion of waves is not normal. In the case of rotating non-magnetized background one case of normally dispersed waves was found whereas in isothermal plasma with constant pressure, no such case is observed. This means that the background pressure ceases normal dispersion of waves.

In the case of rotating magnetized background, there is one case of normal dispersion and two of anomalous dispersion. The case with normal dispersion of waves occurs when the plasma admits the properties of Veselago medium whereas in cold plasma there were two such cases where the plasma shows the properties of this medium. Thus the cold plasma equation of state provides more chances to the fluid to admit the properties of Veselago medium.

It is worth interesting to note that the wave number becomes infinite and consequently waves vanish at the event horizon in all the cases. This corresponds to the well-known fact that no information can be extracted from a black hole. The negative phase velocity propagation regions are found in the cases of rotating background which are discussed by Mackay et al. in 30 according to which rotation of a black hole is required for negative phase velocity propagation. The same results were found in the case of cold plasma. Thus it can be deduced that the rotation brings negative phase velocity whether pressure is involved in the surrounding plasma or not. Mackay et al. 35] found that negative phase velocity propagation was neither observed in Schwarzschild-anti de Sitter spacetime, nor found outside the event horizon of a Schwarzschild black hole due to zero rotation. This is what we have obtained for non-rotating case. Ross et al. [36] also investigated the negative phase velocity propagation phenomenon for Kerr-Newmann and Kerr-Sen metrics particularly close to the outer event horizon when the magnitude of the charge is large.

The complex solutions of the dispersion equation are not included because it is felt that these solutions will prove to be of little significance compared to those which have been discussed. The dispersion relations are obtained using 3+1 ADM formalism and contain the factor of acceleration (depend on lapse function and equals to $-g$ ) which makes them different from the usual MHD dispersion relations. We have investigated the waves propagating in a plasma influenced by the gravitational field. These internal gravity waves interrupt the MHD waves, therefore we have cases of dispersion in each hypersurface. It is mentioned here that some of the figures have patches missing which are due to the existence of complex values there. Mathematica cannot plot complex numbers with real numbers. These complex numbers are shown by the gaps in the figures. It would be interesting to investigate this analysis for the Kerr spacetime. Presently, this work is in progress.

\section{Acknowledgment}

We would like to thank the Higher Education Commission (HEC) Islamabad, Pakistan for the financial support through the Indigenous PhD 5000 Fellowship Program Batch-II.

\section{Appendix A}

This Appendix includes the details to reach at the perturbed form of the GRMHD equations meant for the non-rotating background. The component form of these equations is also given. 
The perturbed flow of isothermal plasma shall be characterized by its fluid density $\rho$, pressure $p$, velocity $\mathbf{V}$ and magnetic field $\mathbf{B}$ (as measured by the FIDO). The first order perturbations in the above mentioned quantities are denoted by $\delta \rho, \delta p, \delta \mathbf{V}$ and $\delta \mathbf{B}$. Consequently, the perturbed variables take the following form

$$
\begin{aligned}
\rho & =\rho^{0}+\delta \rho=\rho^{0}+\rho \tilde{\rho} \\
p & =p^{0}+\delta p=p^{0}+p \tilde{p} \\
\mathbf{B} & =\mathbf{B}^{0}+\delta \mathbf{B}=\mathbf{B}^{0}+B \mathbf{b} \\
\mathbf{V} & =\mathbf{V}^{0}+\delta \mathbf{V}=\mathbf{V}^{0}+\mathbf{v},
\end{aligned}
$$

where $\rho^{0}, p^{0}, \mathbf{B}^{0}$ and $\mathbf{V}^{0}$ are unperturbed quantities. The waves can propagate in $z$-direction due to gravitation with respect to time $t$, hence the perturbed quantities depend on $z$ and $t$.

For the non-rotating background, the perturbed flow of fluid is only along $z$-axis. Thus the FIDOs measured four-velocity and magnetic field are along $z$-axis which can be expressed as $\mathbf{V}=u(z) \mathbf{e}_{\mathbf{z}}, \mathbf{B}=B(z) \mathbf{e}_{\mathbf{z}}$ and the Lorentz factor is $\gamma=\frac{1}{\sqrt{1-u^{2}}}$. The perturbed quantities admit the following notations

$$
\tilde{\rho} \equiv \frac{\delta \rho}{\rho}=\tilde{\rho}(t, z), \quad \tilde{p} \equiv \frac{\delta p}{p}=\tilde{p}(t, z), \quad \mathbf{v} \equiv \delta \mathbf{V}=v_{z}(t, z) \mathbf{e}_{\mathbf{z}}, \quad \mathbf{b} \equiv \frac{\delta \mathbf{B}}{B}=b_{z}(t, z) \mathbf{e}_{\mathbf{z}} .
$$

Introducing perturbations given by Eq.(A1) in the GRMHD Eqs.(2.11)-(2.15), we obtain

$$
\begin{aligned}
& \frac{\partial(\delta \mathbf{B})}{\partial t}=\nabla \times(\alpha \mathbf{v} \times \mathbf{B})+\nabla \times(\alpha \mathbf{V} \times \delta \mathbf{B}), \\
& \nabla \cdot(\delta \mathbf{B})=0, \\
& \frac{\partial(\delta \rho+\delta p)}{\partial t}+(\alpha \mathbf{V} \cdot \nabla)(\delta \rho+\delta p)+(\rho+p) \gamma^{2} \mathbf{V} \cdot \frac{\partial \mathbf{v}}{\partial t} \\
& -\alpha(\rho+p) \mathbf{v} \cdot \nabla \ln u+\alpha(\rho+p)(\nabla \cdot \mathbf{v})+(\delta \rho+\delta p) \nabla \cdot(\alpha \mathbf{V})+(\delta \rho+\delta p) \gamma^{2} \mathbf{V} \cdot(\alpha \mathbf{V} \cdot \nabla) \mathbf{V} \\
& +2(\rho+p) \gamma^{2}(\mathbf{V} \cdot \mathbf{v})(\alpha \mathbf{V} \cdot \nabla) \ln \gamma+(\rho+p) \gamma^{2}(\alpha \mathbf{V} \cdot \nabla \mathbf{V}) \cdot \mathbf{v}+(\rho+p) \gamma^{2} \mathbf{V} \cdot(\alpha \mathbf{V} \cdot \nabla) \mathbf{v}=0, \\
& \left\{\left((\rho+p) \gamma^{2}+\frac{\mathbf{B}^{2}}{4 \pi}\right) \delta_{i j}+(\rho+p) \gamma^{4} V_{i} V_{j}-\frac{1}{4 \pi} B_{i} B_{j}\right\} \frac{1}{\alpha} \frac{\partial v^{j}}{\partial t}+\frac{1}{4 \pi}\left[\mathbf{B} \times\left\{\mathbf{V} \times \frac{1}{\alpha} \frac{\partial(\delta \mathbf{B})}{\partial t}\right\}\right]_{i} \\
& +(\rho+p) \gamma^{2} v_{i, j} V^{j}+(\rho+p) \gamma^{4} V_{i} v_{j, k} V^{j} V^{k}-\frac{1}{4 \pi \alpha}\left\{\left(\alpha \delta B_{i}\right)_{, j}-\left(\alpha \delta B_{j}\right)_{i}\right\} B^{j} \\
& =-\gamma^{2}\left\{(\delta \rho+\delta p)+2(\rho+p) \gamma^{2}(\mathbf{V} \cdot \mathbf{v})\right\} a_{i}-(\delta p), i+\frac{1}{4 \pi \alpha}\left\{\left(\alpha B_{i}\right)_{, j}-\left(\alpha B_{j}\right)_{, i}\right\} \delta B^{j} \\
& -(\rho+p) \gamma^{4}\left(v_{i} V^{j}+v^{j} V_{i}\right) V_{k, j} V^{k}-\gamma^{2}\left\{(\delta \rho+\delta p) V^{j}+2(\rho+p) \gamma^{2}(\mathbf{V} \cdot \mathbf{v}) V^{j}+(\rho+p) v^{j}\right\} V_{i, j} \\
& -\gamma^{4} V_{i}\left\{(\delta \rho+\delta p) V^{j}+4(\rho+p) \gamma^{2}(\mathbf{V} \cdot \mathbf{v}) V^{j}+(\rho+p) v^{j}\right\} V_{j, k} V^{k}, \\
& \gamma^{2} \frac{1}{\alpha} \frac{\partial(\delta \rho+\delta p)}{\partial t}+\frac{2}{\alpha}(\rho+p) \gamma^{4} \mathbf{V} \cdot \frac{\partial \mathbf{v}}{\partial t}-2(\rho+p) \gamma^{4}(\mathbf{V} \cdot \mathbf{v})(\mathbf{V} \cdot \nabla) \ln u+6(\rho+p) \gamma^{6}(\mathbf{V} \cdot \mathbf{v})\{\mathbf{V} \cdot(\mathbf{V} \cdot \nabla) \mathbf{V}\} \\
& +(\rho+p) \gamma^{4} \mathbf{V} \cdot(\mathbf{v} \cdot \nabla) \mathbf{V}+2(\rho+p) \gamma^{4} \mathbf{V} \cdot(\mathbf{V} \cdot \nabla) \mathbf{v}+2(\delta \rho+\delta p) \gamma^{2} \mathbf{V} \cdot \mathbf{a}+2(\rho+p) \gamma^{4}(\mathbf{V} \cdot \mathbf{v})(\mathbf{V} \cdot \mathbf{a}) \\
& +(\rho+p) \gamma^{2}(\nabla \cdot \mathbf{v})+(\rho+p) \gamma^{2} \mathbf{v} \cdot \mathbf{a}+\gamma^{2}(\mathbf{V} \cdot \nabla)(\delta \rho+\delta p)-\frac{1}{\alpha} \frac{\partial(\delta p)}{\partial t}-(\rho+p) \gamma^{2}(\mathbf{v} \cdot \nabla) \ln u \\
& +2(\delta \rho+\delta p) \gamma^{4} \mathbf{V} \cdot(\mathbf{V} \cdot \nabla) \mathbf{V}+(\delta \rho+\delta p) \gamma^{2}(\nabla \cdot \mathbf{V})+2(\rho+p) \gamma^{4}(\mathbf{V} \cdot \mathbf{v})(\nabla \cdot \mathbf{V})+2(\rho+p) \gamma^{4} \mathbf{v} \cdot(\mathbf{V} \cdot \nabla) \mathbf{V} \\
& +\frac{1}{4 \pi \alpha}[(\mathbf{V} \times \mathbf{B}) \cdot(\nabla \times(\alpha \delta \mathbf{B}))+(\mathbf{v} \times \mathbf{B}) \cdot(\nabla \times(\alpha \mathbf{B}))+(\mathbf{V} \times \delta \mathbf{B}) \cdot(\nabla \times(\alpha \mathbf{B})) \\
& \left.+(\mathbf{V} \times \mathbf{B}) \cdot\left(\mathbf{V} \times \frac{\partial \delta \mathbf{B}}{\partial t}\right)+(\mathbf{V} \times \mathbf{B}) \cdot\left(\frac{\partial \mathbf{v}}{\partial t} \times \mathbf{B}\right)\right]=0 .
\end{aligned}
$$

The component form of Eqs. (A2)-

$$
\begin{aligned}
& \frac{1}{\alpha} \frac{\partial b_{z}}{\partial t}=0, \\
& b_{z, z}=0, \\
& \rho \frac{\partial \tilde{\rho}}{\partial t}+u \alpha\left(\rho \tilde{\rho}_{, z}+p \tilde{p}_{, z}\right)+p \frac{\partial \tilde{p}}{\partial t}+\gamma^{2} u(\rho+p) \frac{\partial v_{z}}{\partial t}+\alpha(\rho+p)\left(1+\gamma^{2} u^{2}\right) v_{z, z} \\
& -(\tilde{\rho}-\tilde{p})\left\{(\alpha u p)^{\prime}+\alpha \gamma^{2} u^{2} p u^{\prime}\right\}=\alpha(\rho+p)\left(1-2 \gamma^{2} u^{2}\right)\left(1+\gamma^{2} u^{2}\right) \frac{u^{\prime}}{u} v_{z},
\end{aligned}
$$




$$
\begin{aligned}
& (\rho+p) \gamma^{2}\left(1+\gamma^{2} u^{2}\right)\left\{\frac{1}{\alpha} \frac{\partial v_{z}}{\partial t}+u v_{z, z}\right\}=-(\rho \tilde{\rho}+p \tilde{p}) \gamma^{2}\left\{a_{z}+u\left(1+\gamma^{2} u^{2}\right) u^{\prime}\right\} \\
& \left.-\gamma^{2}(\rho+p)\left\{u^{\prime}\left(1+\gamma^{2} u^{2}\right)\left(1+4 \gamma^{2} u^{2}\right)+2 u \gamma^{2} a_{z}\right)\right\} v_{z}-p \tilde{p}^{\prime}-\tilde{p} p^{\prime}, \\
& \rho \gamma^{2} \frac{1}{\alpha} \frac{\partial}{\partial t} \tilde{\rho}+p \gamma^{2} \frac{1}{\alpha} \frac{\partial}{\partial t} \tilde{p}+\frac{2}{\alpha}(\rho+p) \gamma^{4} u \frac{\partial v_{z}}{\partial t}-p \frac{1}{\alpha} \frac{\partial \tilde{p}}{\partial t}+(\rho \tilde{\rho}+p \tilde{p}) \gamma^{2}\left\{2 u a_{z}+2 \gamma^{2} u^{2} u^{\prime}+u^{\prime}\right\} \\
& +\gamma^{2} u\left(\rho_{, z} \tilde{\rho}+\tilde{\rho}_{, z} \rho+p, z \tilde{p}+\tilde{p}_{, z} p\right)+(\rho+p) \gamma^{2}\left(1+2 \gamma^{2} u^{2}\right) v_{z, z} \\
& +(\rho+p) \gamma^{2}\left\{\left(3 \gamma^{2} u u^{\prime}+a_{z}\right)\left(1+2 \gamma^{2} u^{2}\right)-\frac{u^{\prime}}{u}\right\} v_{z}=0 .
\end{aligned}
$$

The conservation law of rest-mass [21] in three-dimensional hypersurface for isothermal state of plasma $\alpha(\rho+$ p) $\gamma u=$ constant is used to obtain Eqs. (A6) and (A9).

\section{Appendix B}

When we consider non-magnetized isothermal plasma in rotating background, i.e., $\mathbf{B}=\mathbf{0}$, the GRMHD Eqs.(2.11) and (2.12) vanish and Eqs.(2.13)-(2.15) change into general relativistic hydrodynamical (GRHD) equations given as follows

$$
\begin{aligned}
& \frac{\partial(\rho+p)}{\partial t}+(\alpha \mathbf{V} \cdot \nabla)(\rho+p)+(\rho+p) \gamma^{2} \mathbf{V} \cdot \frac{\partial \mathbf{V}}{\partial t}+(\rho+p) \gamma^{2} \mathbf{V} \cdot(\alpha \mathbf{V} \cdot \nabla) \mathbf{V}+(\rho+p) \nabla \cdot(\alpha \mathbf{V})=0, \\
& \left.(\rho+p)\left\{\gamma^{2} \delta_{i j}+\gamma^{4} V_{i} V_{j}\right)\right\}\left(\frac{1}{\alpha} \frac{\partial}{\partial t}+\mathbf{V} \cdot \nabla\right) V^{j}=-(\rho+p) \gamma^{2} a_{i}-p_{, i}, \\
& \gamma^{2}\left(\frac{1}{\alpha} \frac{\partial}{\partial t}+\mathbf{V} \cdot \nabla\right)(\rho+p)+2(\rho+p) \gamma^{4} \mathbf{V} \cdot\left(\frac{1}{\alpha} \frac{\partial}{\partial t}+\mathbf{V} \cdot \nabla\right) \mathbf{V}+2(\rho+p) \gamma^{2} \mathbf{V} \cdot \mathbf{a}-\frac{1}{\alpha} \frac{\partial p}{\partial t} \\
& +(\rho+p) \gamma^{2} \nabla \cdot \mathbf{V}=0 .
\end{aligned}
$$

We assume that the fluid is moving in $x z$-plane hence the fluid measured four-velocity by FIDO can be

written as $\mathbf{V}=V(z) \mathbf{e}_{\mathbf{x}}+u(z) \mathbf{e}_{\mathbf{z}}$ for which the Lorentz factor becomes $\gamma=\frac{1}{\sqrt{1-u^{2}-V^{2}}}$. The following notations will be used for the perturbed quantities

$$
\tilde{\rho} \equiv \frac{\delta \rho}{\rho}=\tilde{\rho}(t, z), \quad \tilde{p} \equiv \frac{\delta p}{p}=\tilde{p}(t, z), \quad \mathbf{v} \equiv \delta \mathbf{V}=v_{x}(t, z) \mathbf{e}_{\mathbf{x}}+v_{z}(t, z) \mathbf{e}_{\mathbf{z}}
$$

Introducing perturbations in Eqs.(B1)-(B3), it follows that

$$
\begin{aligned}
& \frac{\partial(\delta \rho+\delta p)}{\partial t}+(\rho+p) \gamma^{2} \mathbf{V} \cdot \frac{\partial \mathbf{v}}{\partial t}+\alpha(\rho+p) \gamma^{2} \mathbf{V} \cdot(\mathbf{V} \cdot \nabla) \mathbf{v}+\alpha(\rho+p)(\nabla \cdot \mathbf{v}) \\
& +(\delta \rho+\delta p) \nabla \cdot(\alpha \mathbf{V})+(\delta \rho+\delta p) \gamma^{2} \mathbf{V} \cdot(\alpha \mathbf{V} \cdot \nabla) \mathbf{V}+(\alpha \mathbf{V} \cdot \nabla)(\delta \rho+\delta p) \\
& +2 \alpha(\rho+p) \gamma^{2}(\mathbf{V} \cdot \mathbf{v})(\mathbf{V} \cdot \nabla) \ln \gamma+\alpha(\rho+p) \gamma^{2}(\mathbf{V} \cdot \nabla \mathbf{V}) \cdot \mathbf{v}-\alpha(\rho+p)(\mathbf{v} \cdot \nabla \ln u)=0 \\
& \left\{(\rho+p) \gamma^{2} \delta_{i j}+(\rho+p) \gamma^{4} V_{i} V_{j}\right\} \frac{1}{\alpha} \frac{\partial v^{j}}{\partial t}+(\rho+p) \gamma^{2} v_{i, j} V^{j}+(\rho+p) \gamma^{4} V_{i} v_{j, k} V^{j} V^{k} \\
& =-\gamma^{2}\left\{(\delta \rho+\delta p)+2(\rho+p) \gamma^{2}(\mathbf{V} \cdot \mathbf{v})\right\} a_{i}-(\delta p)_{, i}-(\rho+p) \gamma^{4}\left(v_{i} V^{j}+v^{j} V_{i}\right) V_{k, j} V^{k} \\
& -\gamma^{2}\left\{(\delta \rho+\delta p) V^{j}+2(\rho+p) \gamma^{2}(\mathbf{V} \cdot \mathbf{v}) V^{j}+(\rho+p) v^{j}\right\} V_{i, j} \\
& -\gamma^{4} V_{i}\left\{(\delta \rho+\delta p) V^{j}+4(\rho+p) \gamma^{2}(\mathbf{V} \cdot \mathbf{v}) V^{j}+(\rho+p) v^{j}\right\} V_{j, k} V^{k} \\
& \gamma^{2} \frac{1}{\alpha} \frac{\partial(\delta \rho+\delta p)}{\partial t}+\frac{2}{\alpha}(\rho+p) \gamma^{4} \mathbf{V} \cdot \frac{\partial \mathbf{v}}{\partial t}-2(\rho+p) \gamma^{4}(\mathbf{V} \cdot \mathbf{v})(\mathbf{V} \cdot \nabla) \ln u+6(\rho+p) \gamma^{6}(\mathbf{V} \cdot \mathbf{v})\{\mathbf{V} \cdot(\mathbf{V} \cdot \nabla) \mathbf{V}\} \\
& +(\rho+p) \gamma^{4} \mathbf{V} \cdot(\mathbf{v} \cdot \nabla) \mathbf{V}+2(\rho+p) \gamma^{4}\{\mathbf{V} \cdot(\mathbf{V} \cdot \nabla) \mathbf{v}+\mathbf{v} \cdot(\mathbf{V} \cdot \nabla) \mathbf{V}\}+2(\delta \rho+\delta p) \gamma^{2} \mathbf{V} \cdot \mathbf{a} \\
& +2(\rho+p) \gamma^{4}(\mathbf{V} \cdot \mathbf{v})(\mathbf{V} \cdot \mathbf{a})+(\rho+p) \gamma^{2}(\nabla \cdot \mathbf{v})+(\rho+p) \gamma^{2} \mathbf{v} \cdot \mathbf{a}+\gamma^{2}(\mathbf{V} \cdot \nabla)(\delta \rho+\delta p)-\frac{1}{\alpha} \frac{\partial(\delta p)}{\partial t} \\
& -(\rho+p) \gamma^{2}(\mathbf{v} \cdot \nabla) \ln u+2(\delta \rho+\delta p) \gamma^{4} \mathbf{V} \cdot(\mathbf{V} \cdot \nabla) \mathbf{V}+(\delta \rho+\delta p) \gamma^{2}(\nabla \cdot \mathbf{V}) \\
& +2(\rho+p) \gamma^{4}(\mathbf{V} \cdot \mathbf{v})(\nabla \cdot \mathbf{V})=0 .
\end{aligned}
$$


The component form of Eqs.(B4)-(B) is

$$
\begin{aligned}
& \rho \frac{\partial \tilde{\rho}}{\partial t}+p \frac{\partial \tilde{p}}{\partial t}+(\rho+p) \gamma^{2}\left\{V \frac{\partial v_{x}}{\partial t}+u \frac{\partial v_{z}}{\partial t}\right\}+\alpha u \rho \tilde{\rho}_{, z}+\alpha u p \tilde{p}_{, z}+\alpha(\rho+p)\left\{\gamma^{2} V u v_{x, z}+\left(1+\gamma^{2} u^{2}\right) v_{z, z}\right\} \\
& -\frac{1}{\gamma}(\tilde{\rho}-\tilde{p})(\alpha u \gamma p)_{, z}+\alpha(\rho+p) \gamma^{2} u\left\{\left(1+2 \gamma^{2} V^{2}\right) V^{\prime}+2 \gamma^{2} u V u^{\prime}\right\} v_{x} \\
& -\alpha(\rho+p)\left\{\left(1-2 \gamma^{2} u^{2}\right)\left(1+\gamma^{2} u^{2}\right) \frac{u^{\prime}}{u}-2 \gamma^{4} u^{2} V V^{\prime}\right\} v_{z}=0 \\
& (\rho+p) \gamma^{2}\left(1+\gamma^{2} V^{2}\right) \frac{1}{\alpha} \frac{\partial v_{x}}{\partial t}+(\rho+p) \gamma^{4} u V \frac{1}{\alpha} \frac{\partial v_{z}}{\partial t}+(\rho+p) \gamma^{2} u\left(1+\gamma^{2} V^{2}\right) v_{x, z}+(\rho+p) \gamma^{4} u^{2} V v_{z, z} \\
& =-(\rho \tilde{\rho}+p \tilde{p}) \gamma^{2} u\left\{\left(1+\gamma^{2} V^{2}\right) V^{\prime}+\gamma^{2} u V u^{\prime}\right\}-(\rho+p) \gamma^{4} u\left\{\left(1+4 \gamma^{2} V^{2}\right) u u^{\prime}+4 V V^{\prime}\left(1+\gamma^{2} V^{2}\right)\right\} v_{x} \\
& -(\rho+p) \gamma^{2}\left[\left\{\left(1+2 \gamma^{2} u^{2}\right)\left(1+2 \gamma^{2} V^{2}\right)-\gamma^{2} V^{2}\right\} V^{\prime}+2 \gamma^{2}\left(1+2 \gamma^{2} u^{2}\right) u V u^{\prime}\right] v_{z}, \\
& (\rho+p) \gamma^{2}\left(1+\gamma^{2} u^{2}\right)\left(\frac{1}{\alpha} \frac{\partial v_{z}}{\partial t}+u v_{z, z}\right)+(\rho+p) \gamma^{4} u V\left(\frac{1}{\alpha} \frac{\partial v_{x}}{\partial t}+u v_{x, z}\right) \\
& =-(\rho \tilde{\rho}+p \tilde{p}) \gamma^{2}\left\{a_{z}+\left(1+\gamma^{2} u^{2}\right) u u^{\prime}+\gamma^{2} u^{2} V V^{\prime}\right\}-p^{\prime} \tilde{p}-p \tilde{p}_{, z} \\
& -(\rho+p)\left[\gamma^{4}\left\{u^{2} V^{\prime}\left(1+4 \gamma^{2} u^{2}\right)+2 V\left\{a_{z}+\left(1+2 \gamma^{2} u^{2}\right) u u^{\prime}\right\}\right\}\right] v_{x} \\
& -(\rho+p) \gamma^{2}\left[u^{\prime}\left(1+\gamma^{2} u^{2}\right)\left(1+4 \gamma^{2} u^{2}\right)+2 u \gamma^{2}\left\{a_{z}+\left(1+2 \gamma^{2} u^{2}\right) V V^{\prime}\right\}\right] v_{z}, \\
& \gamma^{2} \frac{\rho}{\alpha} \frac{\partial \tilde{\rho}}{\partial t}+\left(\gamma^{2}-1\right) \frac{p}{\alpha} \frac{\partial \tilde{p}}{\partial t}+\frac{2}{\alpha}(\rho+p) \gamma^{4}\left(V \frac{v_{x}}{\partial t}+u \frac{v_{z}}{\partial t}\right)+2(\rho+p) \gamma^{4} u V v_{x, z}+(\rho+p) \gamma^{2}\left(1+2 \gamma^{2} u^{2}\right) v_{z, z} \\
& +\tilde{\rho}\left[2 \rho \gamma^{2} u\left\{a_{z}+\gamma^{2}\left(V V^{\prime}+u u^{\prime}\right)\right\}+\gamma^{2} u \rho^{\prime}\right]+\tilde{p}\left[2 p \gamma^{2} u\left\{a_{z}+\gamma^{2}\left(V V^{\prime}+u u^{\prime}\right)\right\}+\gamma^{2} u p^{\prime}\right] \\
& +\gamma^{2} u \rho \tilde{\rho}, z+\gamma^{2} u^{\prime} \rho \tilde{\rho}+\gamma^{2} u p \tilde{p}, z+\gamma^{2} u^{\prime} p \tilde{p}+2(\rho+p) \gamma^{4}\left\{3 \gamma^{2} u V\left(u u^{\prime}+V V^{\prime}\right)+u V a_{z}+u^{\prime} V\right\} v_{x} \\
& +(\rho+p) \gamma^{2}\left\{6 \gamma^{4} u^{2}\left(V V^{\prime}+u u^{\prime}\right)+\gamma^{2}\left(V V^{\prime}+u u^{\prime}\right)+a_{z}\left(1+2 \gamma^{2} u^{2}\right)-\frac{u^{\prime}}{u}+2 \gamma^{2} u u^{\prime}\right\}=0 .
\end{aligned}
$$

\section{Appendix C}

The GRMHD equations for the rotating magnetized background with isothermal state of plasma remain the same as given by Eqs.(2.11)-(2.15). Hence their perturbed form will remain the same as given in Appendix A (i.e., Eqs.(A2)-(A6)).

In this case, fluid's four-velocity is the same as given in the previous section. The rotating magnetic field can be expressed in $x z$-plane, i.e., $\mathbf{B}=B\left[\lambda(z) \mathbf{e}_{\mathbf{x}}+\mathbf{e}_{\mathbf{z}}\right]$. We shall use the following notations for the perturbed magnetic field in addition to the notations given by Eq.(4.1)

$$
\mathbf{b} \equiv \frac{\delta \mathbf{B}}{B}=b_{x}(t, z) \mathbf{e}_{\mathbf{x}}+b_{z}(t, z) \mathbf{e}_{\mathbf{z}} .
$$

Hence, the component form of Eqs. A2 - (A6) can be written, after a tedious algebra, as follows

$$
\begin{aligned}
& \frac{\partial b_{x}}{\partial t}+\alpha u b_{x, z}=\alpha^{\prime}\left(v_{x}-\lambda v_{z}+V b_{z}-u b_{x}\right)+\alpha\left(v_{x, z}-\lambda v_{z, z}-\lambda^{\prime} v_{z}+V^{\prime} b_{z}+V b_{z, z}-u^{\prime} b_{x}\right) \\
& \frac{\partial b_{z}}{\partial t}+\alpha u b_{z, z}=0 \\
& b_{z, z}=0 \\
& \rho \frac{\partial \tilde{\rho}}{\partial t}+p \frac{\partial \tilde{p}}{\partial t}+(\rho+p) \gamma^{2}\left\{V \frac{\partial v_{x}}{\partial t}+u \frac{\partial v_{z}}{\partial t}\right\}+\alpha u \rho \tilde{\rho}_{, z}+\alpha u p \tilde{p}, z+\alpha(\rho+p)\left\{\gamma^{2} V u v_{x, z}+\left(1+\gamma^{2} u^{2}\right) v_{z, z}\right\} \\
& -\frac{1}{\gamma}(\tilde{\rho}-\tilde{p})(\alpha u \gamma p)_{, z}+\alpha(\rho+p) \gamma^{2} u\left\{\left(1+2 \gamma^{2} V^{2}\right) V^{\prime}+2 \gamma^{2} u V u^{\prime}\right\} v_{x} \\
& -\alpha(\rho+p)\left\{\left(1-2 \gamma^{2} u^{2}\right)\left(1+\gamma^{2} u^{2}\right) \frac{u^{\prime}}{u}-2 \gamma^{4} u^{2} V V^{\prime}\right\} v_{z}=0 \\
& \left\{(\rho+p) \gamma^{2}\left(1+\gamma^{2} V^{2}\right)+\frac{B^{2}}{4 \pi}\right\} \frac{1}{\alpha} \frac{\partial v_{x}}{\partial t}+\left\{(\rho+p) \gamma^{4} u V-\frac{\lambda B^{2}}{4 \pi}\right\} \frac{1}{\alpha} \frac{\partial v_{z}}{\partial t}
\end{aligned}
$$




$$
\begin{aligned}
& +\left\{(\rho+p) \gamma^{2}\left(1+\gamma^{2} V^{2}\right)-\frac{B^{2}}{4 \pi}\right\} u v_{x, z}+\left\{(\rho+p) \gamma^{4} u V+\frac{\lambda B^{2}}{4 \pi}\right\} u v_{z, z}-\frac{B^{2}}{4 \pi}\left(1-u^{2}\right) b_{x, z} \\
& -\frac{B^{2}}{4 \pi \alpha} b_{x}\left\{\alpha^{\prime}\left(1-u^{2}\right)-\alpha u u^{\prime}\right\}+(\tilde{\rho} \rho+p \tilde{p}) \gamma^{2} u\left\{\left(1+\gamma^{2} V^{2}\right) V^{\prime}+\gamma^{2} u V u^{\prime}\right\} \\
& +\left[(\rho+p) \gamma^{4} u\left\{\left(1+4 \gamma^{2} V^{2}\right) u u^{\prime}+4\left(1+\gamma^{2} V^{2}\right) V V^{\prime}\right\}+\frac{B^{2} u \alpha^{\prime}}{4 \pi \alpha}\right] v_{x} \\
& +\left[(\rho+p) \gamma^{2}\left[\left\{\left(1+2 \gamma^{2} u^{2}\right)\left(1+2 \gamma^{2} V^{2}\right)-\gamma^{2} V^{2}\right\} V^{\prime}+2 \gamma^{2}\left(1+2 \gamma^{2} u^{2}\right) u V u^{\prime}\right]+\frac{B^{2} u}{4 \pi \alpha}(\lambda \alpha)^{\prime}\right] v_{z}=0, \\
& \left\{(\rho+p) \gamma^{2}\left(1+\gamma^{2} u^{2}\right)+\frac{\lambda^{2} B^{2}}{4 \pi}\right\} \frac{1}{\alpha} \frac{\partial v_{z}}{\partial t}+\left\{(\rho+p) \gamma^{4} u V-\frac{\lambda B^{2}}{4 \pi}\right\} \frac{1}{\alpha} \frac{\partial v_{x}}{\partial t} \\
& \left.+\left\{(\rho+p) \gamma^{2}\left(1+\gamma^{2} u^{2}\right)-\frac{\lambda^{2} B^{2}}{4 \pi}\right\} u v_{z, z}+\left\{(\rho+p) \gamma^{4} u V+\frac{\lambda B^{2}}{4 \pi}\right) u v_{x, z}\right\}+\frac{\lambda B^{2}}{4 \pi}\left(1-u^{2}\right) b_{x, z} \\
& +\frac{B^{2}}{4 \pi \alpha}\left\{-(\alpha \lambda)^{\prime}+\alpha^{\prime} \lambda-u \lambda(u \alpha)^{\prime}\right\} b_{x}+(\rho \tilde{\rho}+p \tilde{p}) \gamma^{2}\left[a_{z}+u\left\{\left(1+\gamma^{2} u^{2}\right) u^{\prime}+\gamma^{2} u V V^{\prime}\right\}\right] \\
& +\left[(\rho+p) \gamma^{4}\left\{u^{2} V^{\prime}\left(1+4 \gamma^{2} V^{2}\right)+2 V\left(a_{z}+u u^{\prime}\left(1+2 \gamma^{2} u^{2}\right)\right)\right\}+\frac{\lambda B^{2} \alpha^{\prime} u}{4 \pi \alpha}\right] v_{x} \\
& +\left[(\rho+p) \gamma^{2}\left\{u^{\prime}\left(1+\gamma^{2} u^{2}\right)\left(1+4 \gamma^{2} u^{2}\right)+2 u \gamma^{2}\left\{\left(1+2 \gamma^{2} u^{2}\right) V V^{\prime}+a_{z}\right\}\right\}-\frac{\lambda B^{2} u}{4 \pi \alpha}(\alpha \lambda)^{\prime}\right] v_{z}=0, \\
& \gamma^{2} \rho \frac{1}{\alpha} \frac{\partial \tilde{\rho}}{\partial t}+\left(\gamma^{2}-1\right) p \frac{1}{\alpha} \frac{\partial \tilde{p}}{\partial t}+\frac{B^{2}}{4 \pi \alpha}(u \lambda-V)\left(u \frac{\partial b_{x}}{\partial t}-V \frac{\partial b_{z}}{\partial t}+\lambda \frac{\partial v_{z}}{\partial t}-\frac{\partial v_{x}}{\partial t}\right) \\
& +\frac{2}{\alpha}(\rho+p) \gamma^{4}\left(V \frac{\partial v_{x}}{\partial t}+u \frac{\partial v_{z}}{\partial t}\right)+2(\rho+p) \gamma^{4} V u v_{x, z}+(\rho+p) \gamma^{2}\left(1+2 \gamma^{2} u^{2}\right) v_{z, z} \\
& +\tilde{\rho} \gamma^{2}\left[2 \rho u\left\{a_{z}+\gamma^{2}\left(V V^{\prime}+u u^{\prime}\right)\right\}+\rho^{\prime} u+\rho u^{\prime}\right]+\tilde{p} \gamma^{2}\left[2 p u\left\{a_{z}+\gamma^{2}\left(V V^{\prime}+u u^{\prime}\right)\right\}+p^{\prime} u+p u^{\prime}\right] \\
& +\gamma^{2} u \rho \tilde{\rho}_{, z}+\gamma^{2} u p \tilde{p}_{, z}-\frac{B^{2} V}{4 \pi \alpha}(\alpha \lambda)^{\prime} b_{z}+\frac{B^{2} u}{4 \pi \alpha}(\alpha \lambda)^{\prime} b_{x}+\frac{B^{2}(u \lambda-V)}{4 \pi \alpha}\left(\alpha b_{x, z}+\alpha^{\prime} b_{x}\right) \\
& +v_{z}\left[(\rho+p) \gamma^{2}\left\{6 \gamma^{4} u^{2}\left(V V^{\prime}+u u^{\prime}\right)+\gamma^{2}\left(V V^{\prime}+u u^{\prime}\right)+\left(1+2 \gamma^{2} u^{2}\right) a_{z}-\frac{u^{\prime}}{u}+2 \gamma^{2} u u^{\prime}\right\}+\frac{B^{2} \lambda}{4 \pi \alpha}(\alpha \lambda)^{\prime}\right] \\
& +v_{x}\left[(\rho+p) \gamma^{4}\left\{2 u V a_{z}+6 \gamma^{2} u V\left(V V^{\prime}+u u^{\prime}\right)+2 u V^{\prime}\right\}-\frac{B^{2}}{4 \pi \alpha}(\alpha \lambda)^{\prime}\right]=0 .
\end{aligned}
$$

\section{References}

[1] Vachaspati, T., Stojkovic, D. and Krauss, L.M.: Observation of Incipient Black Holes and the Information Loss Problem, accepted for publication in Phys. Rev. D (2007), gr-qc/0609024.

[2] Narayan, R.: New J. Phys. 7(2005)199.

[3] Bekenstein, J.D. and Betchart, G.: Phys. Rev. D74(2006)083009.

[4] Petterson, J.A.: Phys. Rev. D10(1974)3166.

[5] Arnowitt, R., Deser, S. and Misner, C.W.: Gravitation: An Introduction to Current Research (Wiley, New York, 1962).

[6] Hawking, S.W.: Nature 248(1974)30; Commun. Math. Phys. 43(1975)199; Phys. Rev. D13(1976)191.

[7] Hartle, J.B.: Phys. Rev. D8(1973)1010; ibid D9(1974)2749.

[8] Hanni, R.S. and Ruffini R.: Phys. Rev. D8(1973)3259.

[9] Hajicek, P.: Commun. Math. Phys. 36(1974)305.

[10] Znajek, R.L.: Ph.D. Thesis., (University of Cambridge, 1976); Mon. Not. R. Astron. Soc. 185(1978)833. 
[11] Damour, T.: Phys. Rev. D18(1978)3598; Procs. of Second Marcel Grossman Meeting on General Relativity ed. R. Ruffini, North Holland, Amsterdam, (1982)587; Ph.D. Thesis. (University of Paris VI, 1979).

[12] Thorne, K.S. and Macdonald, D.A.: Mon. Not. R. Astron. Soc. 198(1982)339.

[13] Thorne, K.S. and Macdonald, D.A.: Mon. Not. R. Astron. Soc. 198(1982)345.

[14] Thorne, K.S., Price, R.H. and Macdonald, D.A.: Black Holes: The Membrane Paradigm (Yale University Press, New Haven, 1986).

[15] Holcomb, K.A. and Tajima, T.: Phys. Rev. D40(1989)3809.

[16] Holcomb, K.A.: Astrophys. J. 362(1990)381.

[17] Dettmann, C.P., Frankel, N.E. and Kowalenko, V.: Phys. Rev. D48(1993)5655.

[18] Khanna, R.: Mon. Not. R. Astron. Soc. 294(1998)673.

[19] Antón, L., Zantti, O., Miralles, J.A., Martí, J.A., M$M^{\mathbf{A}}$ Ibáñez, J., Font, J.A., Pons, J.A.: Astrophys. J. 637(2006)296.

[20] Komissarov, S.S.: Mon. Not. R. Astron. Soc. 336(2002)759.

[21] Zhang, X.-H.: Phys. Rev. D39(1989)2933.

[22] Zhang, X.-H.: Phys. Rev. D40(1989)3858.

[23] Regge, T. and Wheeler, J.A.: Phys. Rev. 108(1957)1063.

[24] Zerilli, F.: Phys. Rev. D2(1970)2141; J. Math. Phys. 11(1970)2203; Phys. Rev. Lett. 24(1970)737.

[25] Price, R.H.: Phys. Rev. D5(1972)2419; ibid D5(1972)2439.

[26] Wald, R.M.: Phys. Rev. D10(1974)1680.

[27] Sakai, J. and Kawata, T.: J. Phys. Soc. Jpn. 49(1980)747.

[28] Buzzi, V., Hines, K.C. and Treumann, R.A.: Phys. Rev. D51(1995)6663, ibid 51(1995)6677.

[29] Sharif, M. and Sheikh, U.: Cold Plasma Dispersion Relations in the Vicinity of a Schwarzschild Black Hole Horizon, accepted for publication in Gen. Relat. \& Gravit. (2007).

[30] Mackay, T.G., Lakhtakia, A. and Setiawan, S.: New J. Phys. 7(2005)171.

[31] Zel'dovich, Ya. B., and Novikov, I.D.: Uspekhi Fiz. Nauk. 84(1964)377; English translation in Sov. Phys.-Uspekhi, 7(1965)763; Uspekhi Fiz. Nauk. 86(1965)477; English translation in Sov. Phys.-Uspekhi, 8(1966)522.

[32] Jackson, J.D.: Classical Electrodynamics (John Wiley \& Sons, 1999).

[33] Veselago, V.G.: Sov. Phys. Usp. 10(1968)509.

[34] Woodley, J.F. and Mojahedi, M.: Negative Group Velocity in Left-Handed Materials, Antennas and Propagation Society International Symposium and USNC/CNC/URSI National Radio Science Meeting, Columbus, Ohio, USA, June 22-27, 2003, Vol. 4, p 643.

[35] Mackay, T.G., Lakhtakia, A., Setiawan, S.: Europhys. Lett. 71(2005)925.

[36] Ross, B.M., Mackay, T.G., Lakhtakia, A.: Effect of Charge on Negative-Phase-Velocity Propagation of Electromagnetic Waves in the Ergosphere of a Rotating Black Hole, astro-ph/0608412. 